\title{
On the Hamiltonian structure of normal forms at elliptic equilibria of reversible vector fields in $\mathbb{R}^{4}$
}

\author{
Jeroen S.W. Lamb ${ }^{\mathrm{a}, *}$, Mauricio F.S. Lima ${ }^{\mathrm{b}}$, Ricardo M. Martins ${ }^{\mathrm{c}}$, \\ Marco-Antonio Teixeira ${ }^{\mathrm{c}}$, Jiazhong Yang ${ }^{\mathrm{d}}$ \\ ${ }^{a}$ Department of Mathematics, Imperial College London, \\ 180 Queen's Gate, London SW7 2AZ, UK \\ ${ }^{b} C M C C$, UFABC, CEP 09210-580, Santo Andre SP, Brazil \\ ${ }^{c}$ Department of Mathematics, IMECC, UNICAMP, CEP 13083-970, Campinas SP, Brazil \\ ${ }^{d}$ Institute of Mathematics, Peking University, Beijing 100871, China
}

\begin{abstract}
This paper addresses the question whether normal forms of smooth reversible vector fields in $\mathbb{R}^{4}$ at an elliptic equilibrium possess a formal Hamiltonian structure. In the non-resonant case we establish a formal conjugacy between reversible and Hamiltonian normal forms. In the case of non-semi-simple $1: 1$ resonance and $p: q$ resonance with $p+q>2$ we establish a weaker form of equivalence, namely that of a formal orbital equivalence to a Hamiltonian normal form that involves an additional time-reparametrization of orbits. Moreover, in case $p+q>3$ we show that no formal conjugacy to a Hamiltonian normal form exists.
\end{abstract}

Keywords: Normal form, time-reversal symmetry, Hamiltonian, 2010 MSC: 34C20,37G05,37L10,37C15

\section{Contents}

$\begin{array}{lll}1 & \text { Introduction and main result } & 2\end{array}$

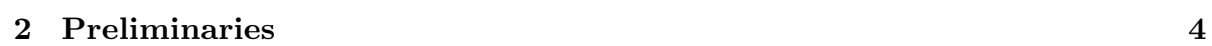

3 Detailed statement of the results $\quad 6$

3.1 Non-resonant case . . . . . . . . . . . . . . . . . . 6

3.2 Non-semi-simple $1: 1$ resonance . . . . . . . . . . . . . . . . . . . . . 7

3.3 1:2 resonance $\ldots \ldots \ldots \ldots \ldots$

$3.4 \quad p: q$ resonance . . . . . . . . . . . . . . . . . . 8

\footnotetext{
* Corresponding author.

Email addresses: jswlamb@imperial.ac.uk (Jeroen S.W. Lamb), mauricio.lima@ufabc.edu.br (Mauricio F.S. Lima), rmiranda@ime.unicamp.br (Ricardo M. Martins), teixeira@ime.unicamp.br (Marco-Antonio Teixeira), jyang@math.pku.edu.cn (Jiazhong Yang)
} 


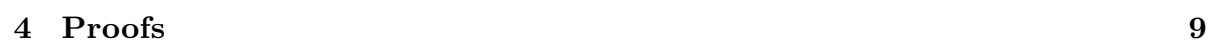

4.1 Non-resonant case $\ldots \ldots \ldots$. . . . . . . . . . . . . . . . . . . . 10

$4.1 .1 \quad$ Formal conjugacy: proof of Theorem 3.1 . . . . . . . . . . . . . 10

4.1.2 Formal orbital equivalence: Theorem 3.2 . . . . . . . . . . . . . 12

4.2 Non-semi-simple 1: 1 resonance: Theorem 3.3 . . . . . . . . . . . . . . 14

$4.3 \quad 1: 2$ resonance: Theorem $3.5 \ldots \ldots \ldots \ldots$

$4.4 \quad p: q$ resonance: Theorem $3.6 \ldots \ldots \ldots \ldots$

\section{Introduction and main result}

The similarity between certain aspects of the dynamical behaviour of timereversible and Hamiltonian dynamical systems, explored already by Poincaré and Birkhoff, has attracted much attention. Many results that hold for Hamiltonian sytems, such as KAM theory and Lyapunov center theorems, have been shown to hold also for time-reversible systems, see for instance [1, 2, 3, 6, 7, 8, 13, 21 and references therein. At the same time, as should be expected, there are also many differences between Hamiltonian and reversible systems, see for instance [13, 20].

In this paper, we address the question whether normal forms of reversible vector fields in $\mathbb{R}^{4}$ at an elliptic equilibrium point formally have a Hamiltonian structure. That is, given a reversible vector field with equilibrium 0 and linear part with two pairs of purely imaginary eigenvalues, we investigate whether there exists a change of coordinates that renders truncations of the Taylor expansion of the vector field at any given order to be Hamiltonian.

The question we address here is antipodal to the one addressed before in [14, 19], concerning the formal reversibility of normal forms at fixed points of symplectic maps of the plane (which turns out to be almost always true). Some semi-global obstructions for reversibility of two-dimensional Hamiltonian vector fields and symplectic diffeomorphisms were presented in [12].

Recall that a vector field $X: \mathbb{R}^{4} \rightarrow \mathbb{R}^{4}$ has a time-reversal symmetry $\varphi \in$ $\operatorname{Diffeo}\left(\mathbb{R}^{4}\right)$ if $\varphi_{*}(X)=-X$, i.e. if $x(t)$ is a solution of $\dot{x}=X(x)$ then so is $\varphi x(-t)$. We also say that $X$ is $\varphi$-reversible, or simply reversible. In this paper we focus on reversible vector fields without additional symmetries and thus assume that $\langle\phi\rangle \simeq \mathbb{Z}_{2}$ (and thus that $\varphi^{2}=\mathrm{id}$ ). In the neighbourhood of a $\varphi$-invariant equilibrium point, say 0 satisfying $X(0)=0$, by Bochner's Theorem [18] it then follows that there exist local coordinates in which $\varphi$ is linear and orthogonal. In this paper we will always assume that we start with such coordinates in the neighbourhood of the equilibrium. From the assumptions on the eigenvalues of the linear part of the vector field we then obtain that near an elliptic equilbrium point $\operatorname{dim} \operatorname{Fix}(\varphi)=2$ where $\operatorname{Fix}(\varphi):=\left\{x \in \mathbb{R}^{4} \mid \varphi(x)=x\right\}$.

A vector field $X$ is called Hamiltonian if there exists a non-degenerate skewsymmetric bilinear form $\omega$ and a function $H: \mathbb{R}^{4} \rightarrow \mathbb{R}$ such that $\omega(X(x), x)=$ $d H(x)$. By the Darboux Theorem, locally (for instance near an equilibrium point) one can always find coordinates such that $X(x)=J \nabla_{x} H(x)$, where

$$
J=\left(\begin{array}{cc}
0 & -I_{2} \\
I_{2} & 0
\end{array}\right),
$$


and $I_{2}$ denotes the $2 \times 2$ unit matrix.

The categories of equivalence that we consider in this paper are formal conjugacy and formal orbital equivalence. We say that two vector fields are formally conjugate if there exists a formal change of coordinates transforming one vector field to the other (to any given order, without concerning the convergence of the transformation in the limit where the order goes to infinity). Two vector fields $X$ and $Y$ are said to be formally orbitally equivalent if there is a smooth function $f: \mathbb{R}^{4} \rightarrow \mathbb{R}$ with no zeros near 0 , so that $f \cdot X$ ( $X$ multiplied by $f$ ) is formally conjugate to $Y$. The multiplication by $f$ has the interpretation of a time-reparametrization of the orbits of $X$. Formal conjugacy between two vector fields implies formal orbital equivalence but not vice versa.

Given a reversible vector field $X$ with equilibrium 0 and derivative $D X(0)$ with eigenvalues $( \pm \alpha i, \pm \beta i)$, we say that the equilibrium of $X$ has a $p: q$ $(p, q \in \mathbb{Z})$ resonance if $q \alpha-p \beta=0$. Without loss of generality we may take $\alpha, \beta>0$ and $\alpha \leq \beta$ so that $p, q \in \mathbb{N}$ with $p \leq q$ and $\operatorname{gcd}(p, q)=1$. Throughout this paper we will assume that vector fields are $C^{\infty}$.

The main results of this paper are summarized in the following theorem:

Theorem 1.1 Let $X$ be a reversible vector field in $\mathbb{R}^{4}$ with equilibrium 0 such that $D X(0)$ has two pairs of purely imaginary eigenvalues $( \pm \alpha i, \pm \beta i)$, with $\alpha, \beta>0$. Then,

(i) If $\alpha: \beta \notin \mathbb{Q}, X$ is generically formally conjugated to an integrable reversible Hamiltonian vector field. Moreover, generically this normal form is formally orbitally five-jet determined: the vector field can be reduced to a polynomial of degree five by the combination of a change of coordinates and rescaling of time.

(ii) If $\alpha: \beta=1: 1$ and $D X(0)$ is not semi-simple (non-semi-simple $1: 1$ resonanc $\left.\S^{1}\right)$ then $X$ is generically not formally conjugate to a Hamiltonian vector field. But $X$ is always formally orbitally equivalent to an integrable Hamiltonian vector field.

(iii) If $\alpha: \beta=1: 2$ then $X$ is formally orbitally equivalent to an integrable Hamiltonian vector field.

(iv) If $\alpha: \beta=p: q \in \mathbb{Q} \backslash\{1: 1,1: 2\}$ ( $p: q$ resonance with $p+q>3$ ) then $X$ is formally orbitally equivalent, but generically not formally conjugate, to a Hamiltonian vector field.

In Section 3 we state more detailed results from which one can immediately deduce the senses of genericity we refer to.

We note that Theorem 1.1 leaves us with one open problem, whether generically the reversible normal form in the case of $1: 2$ resonance is formally

\footnotetext{
1 We note that generically, in one-parameter families of reversible vector fields, nonsemi-simple 1: 1 resonances arise persistenty. The semi-simple 1 : 1 resonance has higher codimension and is not considered here.
} 
conjugate to a Hamiltonian vector field. We have done extensive computerassisted computations to address this problem, but found no persistent counter examples. In particular, in our proof of formal equivalence in the case of $1: 2$ resonance, we show how to begin the conjugacy process up to the third order, and then turn the proof to the equivalence setting for the $k$-jets with $k \geq 4$, due to the apparent lack of freedom while solving some systems.

However, in the presence of additional symmetry (reversing symmetry group $D_{4}$ ) Martins [15, 16 has shown that the formal conjugacy to a Hamiltonian normal form can generically be achieved up to all orders. It is thus tempting to conjecture that formal conjugacy can indeed be obtained generically, but in our experience it is very hard to obtain a proof of this using the methodology set out in this paper.

The non-semi-simple 1: 1 resonant case was considered previously by Van der Meer et al. 17. There, a similarity between the reversible and Hamiltonian cases was found after reduction by the $S^{1}$-equivariance of the normal form that is generated by the semi-simple part of the derivative at $1: 1$ resonance. Our results provide an alternative point of view, and illustrate that the reversible normal form is formally orbitally equivalent to a Hamiltonian vector field.

Similarities between the elliptic points in four-dimensional reversible and Hamiltonian vector fields have also been observed in the context of local bifurcation theory. Indeed, when using Lyapunov-Schmidt reduction, it can be shown that the reduced bifurcation equations for subharmonic branching at $p: q$ resonances in the reversible, Hamiltonian and reversible Hamiltonian contexts are identical, and thus give rise to identical branching patterns of periodic solutions [8]. Our results thus illustrate that the formal equivalence of branching patterns does not imply that the corresponding vector fields are formally conjugate.

Finally, we note that it is of interest to address the question of this paper also in the opposite direction, namely if the normal form of an elliptic equilbrium of a Hamiltonian vector field in $\mathbb{R}^{4}$ is formally reversible. This clearly holds in the non-resonant case (as in the simpler case in $\mathbb{R}^{2}$ ), but in the presence of resonances this problem is still open.

\section{Preliminaries}

In this section we recall some general results and techniques for the normalization of vector fields near an equilibrium solution.

Consider a vector field $X$ on $\mathbb{R}^{n}$ with equilibrium 0 . We are interested in establising coordinates in terms of which (finite order truncations of ) Taylor expansions have special properties. We recall that the existence of a coordinate transformations between two vector fields is a conjugacy relation between these two vector fields. We say that two smooth vector fields $X$ and $Y$ are formally conjugate if for each order $k \geq 1$ there exists a coordinate transformation $\phi$ such that the vector field $X$ and vector field $\phi_{*}(Y)$ (conjugate to $Y$ ) have the same Taylor series expansion up to degree $k$. It is well known that a formal conjugacy does not always imply a true conjugacy, due to the possibility of divergence of the coordinate transformations as $k \rightarrow \infty$. 
Our starting point will often be the well-established result that coordinates can always be chosen in such a way that the nonlinear terms of a finite order Taylor expansion of the vector field commute with the transpose of the linear part of the vector field. This implies in particular that the resulting Taylor expansion commutes with the (closure of) the group generated by the semisimple part (in the sense of Jordan-Chevalley decomposition) of the linear part of the vector field.

Let $L=D X(0)$ denote the linear part of the vector field $X$. Then the Jordan-Chevalley decomposition theorem asserts that $J$ can be written uniquely as the sum $L=S+N$ where $S$ is semi-simple, $N$ is nilpotent and $[S, N]=0$.

The following normal form theorem is a reversible version of a classical result by Belitskii 4 .

Theorem 2.1 ([11]) Let $X$ be a $\varphi$-reversible vector field, where $\varphi$ acts linearly and $\varphi^{2}=\mathrm{Id}$. Let moreover $X$ have equilibrium 0 and linear part $L=D X(0)$ with Jordan-Chevalley decomposition $L=S+N$. Then $X$ is formally conjugate, by a $\varphi$-equivarant coordinate transformation, to a $\varphi$-reversible vector field $\tilde{X}$ with linear part $L$, satisfying $\left[(\tilde{X}-L), L^{T}\right]=0$, where $L^{T}$ denotes the transpose of $L$.

Corollary 2.2 (Formal normal form symmetry) The normal form $\tilde{X}$ from Theorem 2.1 is reversible-equivariant with respect to the group $G \rtimes \mathbb{Z}_{2}(\varphi)$ with $G=\overline{\{\exp (S t) \mid t \in \mathbb{R}\}}$.

The proof of Theorem 2.1 relies on the analysis of the effect of coordinate transformations that are derived from the flow of a vector field.

Assume that $X$ and $Y$ are vector fields such that

$$
j^{k}[X, Y]=0, \quad \text { and } \quad j^{1} Y=0,
$$

where $[X, Y]$ denotes the Lie bracket of $X$ and $Y$, and $j^{k} X$ denotes the $k$-jet of $X$ (equivalence class of vector fields with the same $k$ th order Taylor expansion as $X)$. Let $\phi_{Y}^{t}$ denote the time- $t$ flow of $Y$ and $\tilde{X}=\left(\phi_{Y}^{t}\right)_{*} X$, then we have

$$
j^{k+1}(\tilde{X})=j^{k+1} X+t j^{k+1}[X, Y] .
$$

This implies that if $X$ and $\tilde{X}$ are vector fields such that $j^{k} X=j^{k} \tilde{X}$ and

$$
j^{k+1}[X, Y]=j^{k+1}(\tilde{X}-X)
$$

is solvable with respect to a vector field $Y$ such that $j^{1} Y=0$, then there is a diffeomorphism $\phi$ such that

$$
j^{k+1} \phi_{*} X=j^{k+1} \tilde{X} .
$$

For normalization purposes we usually assume that the linear part of the vector field has been normalized (often to Jordan normal form), and focus on coordinate transformations with linear part the identity (which are formally identical to the time-one maps of flows of vector fields). 
In this paper we carefully examine how within the above framework we can find $Y$ so that $j^{k+1} \tilde{X}$ is Hamiltonian, which requires an effort that goes well beyond the proof of Theorem 2.1, where at each order $k$ it suffices to consider only vector fields $Y$ that are homogeneous of degree $k$.

Since there also exists a (reversible-equivariant) Hamiltonian version of Theorem 2.1 we can moreover assume without loss of generality that $Y$ is $G \rtimes \mathbb{Z}_{2}(\varpi)$ equivariant. Namely, if we would find $Y$ without these properties we could obtain an additional normalization hat preserves the Hamiltonian structure to yield a $G \rtimes \mathbb{Z}_{2}(\varphi)$ reversible-equivariant (and Hamiltonian) normal form. It then follows [11] that such a normal form can also be obtained by a symmetry preserving $\left(G \rtimes \mathbb{Z}_{2}(\varpi)\right.$ equivariant) coordinate transformation.

\section{Detailed statement of the results}

In this section, we present in more detail the results summarized in Theorem 1.1. In each individual case we identify explicitly the Hamiltonian structure of the normal forms. We will often use the identification $\mathbb{C}^{2} \cong \mathbb{R}^{4}$, in coordinates $z_{j}=x_{j}+i y_{j}, j=1,2$. The starting point of our study is usually the normal form of Theorem 2.1, that is characterized by the fact that the nonlinear terms commute with the transpose of the linear part of the vector field. By Corollary 2.2 this implies that this normal form is equivariant with respect to the (Lie) group generated by the semi-simple part of the linear part of the vector field, yielding in the context of this paper a formal symmetry group of the form $S^{1} \times S^{1}$ (in the non-resonance case) or $S^{1}$ (in the case of $p: q$ resonance). Because of this symmetry, it is useful to introduce the variables $A:=z_{1} \overline{z_{1}}$, $B:=z_{2} \overline{z_{2}}, C:=z_{1}^{q}{\overline{z_{2}}}^{p}$ and $D:=\bar{C}$ (which are invariant under the relevant group actions).

\subsection{Non-resonant case}

Let $X$ be a $\varphi$-reversible vector field, with $\varphi\left(z_{1}, z_{2}\right)=\left(i \bar{z}_{1},-i \bar{z}_{2}\right),\left(z_{1}, z_{2}\right) \in$ $\mathbb{C}^{2}$, and $X(0)=0$, so that $D X(0)$ has eigenvalues $( \pm \alpha i, \pm \beta i)$, with $\alpha: \beta \notin \mathbb{Q}$. Then our starting point is the reversible-equivariant normal form

$$
X=\left(\alpha i z_{1}+i z_{1} \sum_{j+l=1}^{\infty} a_{j, l} A^{j} B^{l}\right) \frac{\partial}{\partial z_{1}}+\left(\beta i z_{2}+i z_{2} \sum_{j+l=1}^{\infty} b_{j, l} A^{j} B^{l}\right) \frac{\partial}{\partial z_{2}},
$$

where due to the $\varphi$-reversibility all parameters $a_{j, l}$ and $b_{j, l}$ are real. It turns out that the sign of $a_{0,1} b_{1,0}$ is invariant under changes of coordinates that do not change the linear part of the vector field.

Theorem 3.1 (Non-resonant formal conjugacy) Let $X$ be $\varphi$-reversible and non-resonant, as detailed above. If, with refererence to (3.1), $a_{0,1} b_{1,0} \neq 0$, then $X$ is formally conjugate to a $\varphi$-reversible Hamiltonian vector field with symplectic form $\omega\left(z_{1}, z_{2}\right)=d z_{1} \wedge \varepsilon d z_{2}$, with $\varepsilon:=\operatorname{sgn}\left(a_{0,1} b_{1,0}\right)$, and Hamiltonian $H=\alpha A+\varepsilon \beta B+h(A, B)$, where $h(0,0)=\partial_{A} h(0,0)=\partial_{B} h(0,0)=0$. 
In the orbital equivalence setting, the sign of $a_{0,1} b_{1,0}$ is no longer invariant. Nevertheless, it turns out that, in terms of (3.1), the inequalities

$$
a_{1,0} \beta-b_{1,0} \alpha \neq 0, \quad a_{0,1} \beta-b_{0,1} \alpha \neq 0
$$

are invariant.

Theorem 3.2 (Non-resonant orbital equivalence) Let $X$ be a $\varphi$-reversible vector field given by (3.1) where $\alpha$ and $\beta$ satisfy (3.2). Then $X$ is formally orbitally equivalent to a $\phi$-reversible Hamiltonian vector field with symplectic form $\omega=d z_{1} \wedge d z_{2}$ and Hamiltonian $H=\frac{\alpha}{2} A+\frac{\beta}{2} B+a \frac{A^{2}}{2}+b \frac{B^{2}}{2}+c \frac{A^{3}}{6}$. where $a= \pm 1, b= \pm 1$, and $c \in \mathbb{R}$.

\subsection{Non-semi-simple 1: 1 resonance}

Our starting point is the $S^{1}$-equivariant reversible Belitskii normal form

$$
\begin{aligned}
X= & \left(\alpha i z_{1}+z_{2}+z_{1} f_{1}(A, B, C, D)+z_{2} f_{2}(A, B, C, D)\right) \frac{\partial}{\partial z_{1}}+ \\
& \left(\alpha i z_{2}+z_{1} g_{1}(A, B, C, D)+z_{2} g_{2}(A, B, C, D)\right) \frac{\partial}{\partial z_{2}}
\end{aligned}
$$

where $f_{j}$ and $g_{j}, j=1,2$, have no constant or linear parts. The functions $f$ and $g$ moreover satisfy some conditions imposed by the $\varphi$-reversibility (where without loss of generality we take $\varphi$ as in the non-resonant case), see 4.2 .

Theorem 3.3 Let $X$ be a 1:1 non-semi-simple resonant reversible vector field. Then its normal form is formally orbitally equivalent to a Hamiltonian vector field with symplectic form $\omega$ and Hamiltonian $H=\left(y_{1}^{2}+y_{2}^{2}\right) / 2+v+f(A, v)$, where $v=x_{1} y_{2}-x_{2} y_{1}, A=x_{1}^{2}+x_{2}^{2}$, and $f$ satisfies $f(0,0)=\partial_{A} f(0,0)=$ $\partial_{v} f(0,0)=0$.

However, the normal form is generically not formally conjugate to a Hamiltonian vector field.

Remark 3.4 To be more precise about the final claim of Theorem 3.3, let us give a simple computational argument. Consider the 3-jet of equation (3.3), reduced by the Belitiskii normal form and reversibility. We can write this system in real coordinates as

$$
\left\{\begin{array}{l}
\dot{x}_{1}=-x_{2}+y_{1}-x_{2}\left(a_{1} A+a_{2} v\right) \\
\dot{x}_{2}=x_{1}+y_{2}+x_{1}\left(a_{1} A+a_{2} v\right) \\
\dot{y}_{1}=-y_{2}-y_{2}\left(a_{1} A+a_{2} v\right)+x_{1}\left(a_{3} v+a_{4} A\right) \\
\dot{y}_{2}=y_{1}+y_{1}\left(a_{1} A+a_{2} v\right)+x_{2}\left(a_{3} v+a_{4} A\right),
\end{array}\right.
$$

where $A=x_{1}^{2}+x_{2}^{2}$ and $v=x_{1} y_{2}-x_{2} y_{1}$. Define $H=H_{2}+H_{3}+H_{4}$ and $Y=$ $J \nabla H$, where $H_{k}$ is a homogeneous polynomial of degree $k$ and $J$ is the canonical sympletic matrix. If $X$ is conjugate to some Hamiltonian vector field, then there exist such $H$ and a change of coordinates $\Psi=I d+\psi$, with $\psi=o\left(|x|^{3}\right)$, such that

$$
j^{3}(D \Psi(x) X(x))=j^{3}(Y(\Psi(x)) .
$$


In turns out that a solution can be obtained if and only if $a_{3}=-2 a_{1}$. If this condition holds, at the next (5th) order a similar condition arises.

\subsection{1:2 resonance}

Let $X$ be a $\varphi$-reversible vector field with equilibrium 0 and linear part that has eigenvalues $( \pm \lambda i, \pm 2 \lambda i)$, with $\varphi\left(z_{1}, z_{2}\right)=-\left(\bar{z}_{1}, \bar{z}_{2}\right)$. Then the corresponding Poincaré-Dulac normal form can be written in the form

$$
\left\{\begin{array}{l}
\dot{z}_{1}=i \lambda z_{1}+z_{1} f_{1}(A, B, C, D)+\bar{z}_{1} z_{2} f_{2}(B, D) \\
\dot{z}_{2}=2 i \lambda z_{2}+z_{2} g_{1}(A, B, C, D)+z_{1}^{2} g_{2}(A, C),
\end{array}\right.
$$

where $A=z_{1} \bar{z}_{1}, B=z_{2} \bar{z}_{2}, C=z_{1}^{2} \bar{z}_{2}$, and $D=\bar{C}$.

We are considering the following generical (mutually exclusively satisfied) conditions:

$$
\begin{aligned}
& f_{2}(0,0) g_{2}(0,0)<0 \\
& f_{2}(0,0) g_{2}(0,0)>0
\end{aligned}
$$

The $1: 2$ resonance is unique among other resonances that it has quadratic terms appearing in the preliminary normal form. Since, as a general rule, the lower order a nonlinear resonant term has, the more it influences the local dynamical properties, the appearance of quadratic resonant terms in the $1: 2$ resonance normal form is of particular importance. In applications, the $1: 2$ resonance is often described as being the most energetic, see for instance [5] and references therein for more details.

Theorem 3.5 Let $X$ be a $\varphi$-reversible vector field with equilibrium 0 and $D X(0)$ having purely imaginary eigenvalues at $1: 2$ resonance. Then, with reference to (3.5), if $f_{2}(0,0) g_{2}(0,0)<0$ then $X$ is formally orbitally equivalent to the Hamiltonian vector field

$$
\dot{z}_{1}=i \frac{\partial H}{\partial \bar{z}_{1}}, \quad \dot{z}_{2}=i \frac{\partial H}{\partial \bar{z}_{2}},
$$

where $H=\lambda(A+2 B)+(C-D) \eta_{1}(A, B)+\eta_{2}(A, B)$.

If $f_{2}(0,0) g_{2}(0,0)>0$ then $X$ is formally orbitally equivalent to

$$
\dot{z}_{1}=i \frac{\partial H}{\partial \bar{z}_{1}}, \quad \dot{z}_{2}=-i \frac{\partial H}{\partial \bar{z}_{2}},
$$

where $H=\lambda(A-2 B)+(C-D) \eta_{1}(A, B)+\eta_{2}(A, B)$.

In either case, $\eta_{1}$ has no constant part and $\eta_{2}$ has no constant or linear part.

\section{4. $p: q$ resonance}

In this section we illustrate the results concerning all the other possible resonant cases. These mainly include $1: N(N \geq 3)$ resonances and $p: q$ $(p>1)$ resonances. We shall not distinguish $1: N$ resonance from $p: q$ although conventionally $p: q$ resonance refers to $p>1$. 
Let $X$ be a $\varphi$ time reversible vector field with $p: q$ resonance. Assume that $\varphi$ takes the form $\varphi\left(z_{1}, z_{2}\right)=-\left(\bar{z}_{1}, \bar{z}_{2}\right)$, then $X$ can be put into the resonant normal form:

$$
\left\{\begin{array}{l}
\dot{z}_{1}=p i \lambda z_{1}+z_{1} f_{1}(A, B, C, D)+\bar{z}_{1}^{q-1} z_{2}^{p} f_{2}(A, B, C, D) \\
\dot{z}_{2}=q i \lambda z_{2}+z_{2} g_{1}(A, B, C, D)+z_{1}^{q} \bar{z}_{2}^{p-1} g_{2}(A, B, C, D),
\end{array}\right.
$$

where $A=z_{1} \bar{z}_{1}, B=z_{2} \bar{z}_{2}, C=z_{1}^{q} \bar{z}_{2}^{p}, D=\bar{C}$.

As mentioned previously, all resonant terms in (3.7) are derived from two kinds of terms. One is from $z_{j} A^{m} B^{n}$, and the other is from $\bar{z}_{1}^{q-1} z_{2}^{p} \partial / \partial z_{1}$ and $z_{1}^{q} \bar{z}_{2}^{p-1} \partial / \partial z_{2}$. Respectively, we call them the first and the second kind of resonant terms. The lowest order of the first kind of resonant terms is 3 whereas the lowest order of the second kind of resonant terms is $p+q-1$ which is no less than 3 . Note that the the sign of $f_{2}(0,0,0,0) g_{2}(0,0,0,0)$ is invariant under changes of coordinates and the multiplication of real functions. It is also interesting to note that the symplectic forms depend only on the second kind of resonant terms with the lowest order (see [5] and reference therein for more details on the dynamics of the lowest order terms). Thus generically we have two cases $f_{2}(0,0,0,0) g_{2}(0,0,0,0)>0$ and $f_{2}(0,0,0,0) g_{2}(0,0,0,0)<0$.

In this paper, we prove the following

Theorem 3.6 Let $X$ be a $\varphi$ time reversible vector field having $p: q$ resonance and having a generic nonlinear part. In terms of (3.7), if $f_{2}(0,0,0,0) g_{2}(0,0,0,0)<$ 0 , then $X$ is formally orbitally equivalent to a Hamiltonian vector field

$$
\dot{z}_{1}=i \frac{\partial H}{\partial \bar{z}_{1}}, \quad \dot{z}_{2}=i \frac{\partial H}{\partial \bar{z}_{2}},
$$

where $H=p z_{1} \bar{z}_{1}+q z_{2} \bar{z}_{2}+\cdots$. If $f_{2}(0,0,0,0) g_{2}(0,0,0,0)>0$, then $X$ is formally orbitally equivalent to a Hamiltonian vector field

$$
\dot{z}_{1}=i \frac{\partial H}{\partial \bar{z}_{1}}, \quad \dot{z}_{2}=-i \frac{\partial H}{\partial \bar{z}_{2}},
$$

where $H=p z_{1} \bar{z}_{1}-q z_{2} \bar{z}_{2}+\cdots$ and the dots denote the higher order terms. Moreover, generically the normal form is not formally conjugate to a Hamiltonian vector field.

\section{Proofs}

In this section we present the proofs for the results stated in Section 3 , and summarized in Theorem 1.1 . 


\subsection{Non-resonant case}

\subsubsection{Formal conjugacy: proof of Theorem 3.1}

We only consider the case $a_{01} b_{10}>0$. The proof in case $a_{01} b_{10}<0$ is similar.

To prove the theorem we need to show that for any fixed $k \geq 0$,

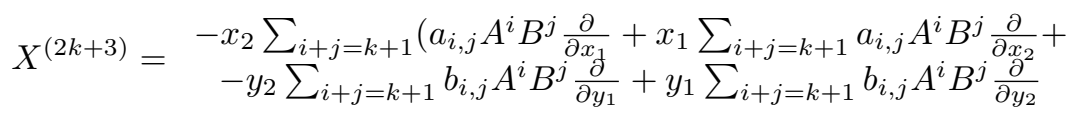

can be so normalized that the following compatibility relations hold.

$$
(j+1) a_{k-j, j+1}=(k-j+1) b_{k-j+1, j}, \quad j=0,1, \ldots, k .
$$

Namely, if these compatibility conditions are satisfied then there exists a generating function of the form $H_{k}=\sum_{i+j=k+2} h_{i, j} A^{i} B^{j}$, where $h_{i, j}$ will be choosen in an adequate way.

We prove things order-by-order. As the lowest order of resonant terms, $X^{(3)}$ can be normalized to satisfy (4.1). Namely, in terms of (3.1), we can put $a_{0,1}=b_{1,0}$ by applying linear scalings of $z_{1}$ and $z_{2}$, recalling the assumption that $a_{0,1} b_{1,0}>0$. Moreover, one can scale variables so that $a_{0,1}=b_{1,0}= \pm 1$.

Next we show that the normalization of $X^{(2 k+3)}$ can be done for any $k>0$. Following the methodology of Section 2 we have to show the solvability of $Y$ from the following homological equation

$$
\left[X^{(3)}, Y\right]=\tilde{X}^{(2 k+3)}-X^{(2 k+3)},
$$

where $\tilde{X}=J \nabla H_{k}$ for some polynomial Hamiltonian $H_{k}: \mathbb{R}^{4} \rightarrow \mathbb{R}$ of degree $2 k+4$.

Note that

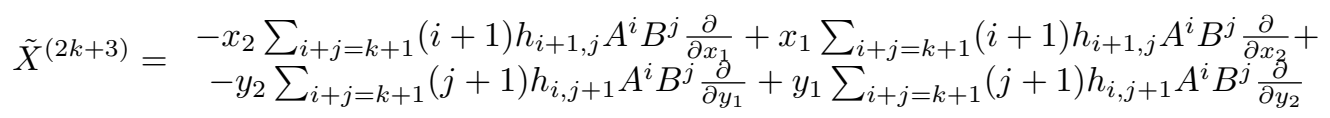

Writing $Y$ in the form

$$
Y=\left(\begin{array}{c}
x_{1}\left(\alpha_{k, 0} A^{k}+\alpha_{k-1,1} A^{k-1} B+\ldots+\alpha_{1, k-1} A B^{k-1}+\alpha_{0, k} B^{k}\right) \\
x_{2}\left(\alpha_{k, 0} A^{k}+\alpha_{k-1,1} A^{k-1} B+\ldots+\alpha_{1, k-1} A B^{k-1}+\alpha_{0, k} B^{k}\right) \\
y_{1}\left(\beta_{k, 0} A^{k}+\beta_{k-1,1} A^{k-1} B+\ldots+\beta_{1, k-1} A B^{k-1}+\beta_{0, k} B^{k}\right) \\
y_{2}\left(\beta_{k, 0} A^{k}+\beta_{k-1,1} A^{k-1} B+\ldots+\beta_{1, k-1} A B^{k-1}+\beta_{0, k} B^{k}\right)
\end{array}\right)
$$

the left side of 4.2 can be calculated explicitly:

$$
\left[X^{(3)}, Y\right]=\left(\begin{array}{c}
-2 x_{2}\left(\gamma_{0} A^{k+1}+\gamma_{1} A^{k} B+\ldots+\gamma_{k} A B^{k}+\gamma_{k+1} B^{k+1}\right) \\
2 x_{1}\left(\gamma_{0} A^{k+1}+\gamma_{1} A^{k} B+\ldots+\gamma_{k} A B^{k}+\gamma_{k+1} B^{k+1}\right) \\
-2 y_{2}\left(\delta_{0} A^{k+1}+\delta_{1} A^{k} B+\ldots+\delta_{k} A B^{k}+\delta_{k+1} B^{k+1}\right) \\
2 y_{1}\left(\delta_{0} A^{k+1}+\delta_{1} A^{k} B+\ldots+\delta_{k} A B^{k}+\delta_{k+1} B^{k+1}\right)
\end{array}\right)
$$

where $\gamma_{0}=\alpha_{k, 0} a_{1,0}, \gamma_{k+1}=\beta_{k, 0} a_{0,1}, \delta_{0}=\alpha_{k, 0} a_{0,1}, \delta_{k+1}=\beta_{0, k} b_{0,1}$ and $\gamma_{j}=$ $\alpha_{k-j, j} a_{1,0}+\beta_{k-j+1, j-1} a_{0,1}, \delta_{j}=\alpha_{k-j, j} a_{0,1}+\beta_{k-j+1, j-1} b_{0,1}$ for $j=1, \ldots, k$. 
The right side of 4.2 is:

$$
\tilde{X}^{(2 k+3)}-X^{(2 k+3)}=\left(\begin{array}{c}
-x_{2}\left(\sum_{i+j=k+1}\left[(i+1) h_{i+1, j}-a_{i, j}\right] A^{i} B^{j}\right) \\
x_{1}\left(\sum_{i+j=k+1}\left[(i+1) h_{i+1, j}-a_{i, j}\right] A^{i} B^{j}\right) \\
-y_{2}\left(\sum_{i+j=k+1}\left[(j+1) h_{i, j+1}-b_{i, j}\right] A^{i} B^{j}\right) \\
y_{1}\left(\sum_{i+j=k+1}\left[(j+1) h_{i, j+1}-b_{i, j}\right] A^{i} B^{j}\right)
\end{array}\right)
$$

Now comparing 4.3 and 4.4 , we see that to solve $\left[X^{(3)}, Y\right]=\tilde{X}^{(2 k+3)}-$ $X^{(2 k+3)}$ is equivalent to solve, for $\alpha$ 's, $\beta$ 's and $h$ 's, the following system of equations:

$$
\left\{\begin{array}{lll}
2 \alpha_{k, 0} a_{1,0} & = & (k+2) h_{k+2,0}-a_{k+1,0} \\
2 \beta_{k, 0} a_{0,1} & = & h_{1, k+1}-a_{0, k+1} \\
2 \alpha_{k, 0} a_{0,1} & = & h_{k+1,1}-b_{k+1,0} \\
2 \beta_{0, k} b_{0,1} & = & (k+2) h_{0, k+2}-b_{0, k+1} \\
2\left[\alpha_{k-1,1} a_{1,0}+\beta_{k, 0} a_{0,1}\right] & = & (k+1) h_{k+1,1}-a_{k, 1} \\
& \vdots \\
2\left[\alpha_{0, k} a_{1,0}+\beta_{1, k-1} a_{0,1}\right] & =2 h_{2, k}-a_{1, k} \\
2\left[\alpha_{k-1,1} a_{0,1}+\beta_{k, 0} b_{0,1}\right] & =2 h_{k, 2}-b_{1, k} \\
& \vdots \\
2\left[\alpha_{0, k} a_{0,1}+\beta_{1, k-1} b_{0,1}\right] & =(k+1) h_{k-1,2}-b_{k, 1}
\end{array}\right.
$$

We rewrite this system as

$$
\begin{cases}2 \alpha_{k, 0} a_{1,0} & =(k+2) h_{k+2,0}-a_{k+1,0} \\ 2 \alpha_{k, 0} a_{0,1} & =h_{k+1,1}-b_{k+1,0} \\ 2 \beta_{k, 0} a_{0,1} & =h_{1, k+1}-a_{0, k+1} \\ 2 \beta_{0, k} b_{0,1} & =(k+2) h_{0, k+2}-b_{0, k+1} \\ 2\left[\alpha_{k-1,1} a_{1,0}+\beta_{k, 0} a_{0,1}\right] & =(k+1) h_{k+1,1}-a_{k, 1} \\ 2\left[\alpha_{k-1,1} a_{0,1}+\beta_{k, 0} b_{0,1}\right] & =2 h_{k, 2}-b_{1, k} \\ & \vdots \\ 2\left[\alpha_{0, k} a_{1,0}+\beta_{1, k-1} a_{0,1}\right] & =2 h_{2, k}-a_{1, k} \\ 2\left[\alpha_{0, k} a_{0,1}+\beta_{1, k-1} b_{0,1}\right] & =(k+1) h_{k-1,2}-b_{k, 1}\end{cases}
$$

As $a_{0,1} \neq 0$, the above system has solution. For $k=1$, one solution is:

$$
\begin{aligned}
\alpha_{1,0} & =1 / 4\left(-2 b_{2,0} a_{0,1}+a_{1,1} a_{0,1}-a_{1,0} b_{1,1}+2 a_{1,0} a_{0,2}\right) / a_{0,1}^{2}, \\
\alpha_{0,1} & =1 / 2\left(2 a_{0,2}-b_{1,1}\right) / a_{0,1}, \\
h_{3,0} & =1 / 12\left(-2 a_{1,0} b_{2,0} a_{0,1}+a_{1,0} a_{1,1} a_{0,1}-a_{1,0}^{2} b_{1,1}+2 a_{1,0}^{2} a_{0,2}+2 a_{2,0} a_{0,1}^{2}\right) / a_{0,1}^{2}, \\
h_{2,1} & =1 / 4\left(a_{1,1} a_{0,1}-a_{1,0} b_{1,1}+2 a_{1,0} a_{0,2}\right) / a_{0,1}, \\
h_{1,2} & =1 / 2 a_{0,2}, \\
h_{0,3} & =1 / 6 b_{0,2} .
\end{aligned}
$$




\subsubsection{Formal orbital equivalence: Theorem 3.2}

Formal conjugacy, as established in the previous section, implies formal orbital equivalence. It remains to be shown that in the formal orbital equivalence setting the resulting Hamiltonian can generically be chosen to be polynomial . The aim is to show that by a combination of a coordinate transformation and multiplication of the vector field by a formal power series with no zeros near 0 , (3.1) can be normalized to a Hamiltonian vector field, with $H$ given in the statement of Theorem 3.2 and $X_{H}$ given by

$$
\left\{\begin{array}{l}
\dot{x}_{1}=-\alpha x_{2}-a x_{2} \Delta_{1}-c x_{2} \Delta_{1}^{2} \\
\dot{x}_{2}=-\alpha x_{1}-a x_{1} \Delta_{1}-c x_{1} \Delta_{1}^{2} \\
\dot{y}_{1}=-\beta y_{2}-b y_{2} \Delta_{2} \\
\dot{y}_{2}=\beta y_{1}+b y_{1} \Delta_{2}
\end{array}\right.
$$

where $a= \pm 1, b= \pm$ and $c \in \mathbb{R}$.

We proceed order by order. We start multiplying the 3 -jet $j^{3} X$ of $X$ by the function

$$
h_{1}=1-\frac{b_{1,0}}{\beta} A-\frac{a_{0,1}}{\alpha} B
$$

yielding

$$
\begin{aligned}
j^{3}\left(h_{1} \cdot X\right) & =\left(-\alpha x_{2}-x_{2} \tilde{a}_{1,0} A\right) \frac{\partial}{\partial x_{1}}+\left(\alpha x_{1}+x_{1} \tilde{a}_{1,0} A\right) \frac{\partial}{\partial x_{2}} \\
& +\left(-\beta y_{2}-y_{2} \tilde{b}_{0,1} B\right) \frac{\partial}{\partial y_{1}}+\left(\beta y_{1}+y_{1} \tilde{b}_{0,1} B\right) \frac{\partial}{\partial y_{1}}
\end{aligned}
$$

where $\tilde{a}_{1,0}=\left(\frac{\beta a_{1,0}-b_{1,0} \alpha}{\beta}\right)$ and $\tilde{b}_{0,1}=\left(\frac{\alpha b_{0,1}-a_{0,1} \beta}{\alpha}\right)$.

Moreover, due to the genericity conditions 3.2 , we can scale $\tilde{a}_{1,0}$ and $\tilde{b}_{0,1}$ to \pm 1 . We thus may normalize $j^{3} X$ to

$$
\left(-\alpha x_{2}-x_{2} \varepsilon_{1} A\right) \frac{\partial}{\partial x_{1}}+\left(\alpha x_{1}+x_{1} \varepsilon_{1} A\right) \frac{\partial}{\partial x_{2}}+\left(-\beta y_{2}-y_{2} \varepsilon_{2} B\right) \frac{\partial}{\partial y_{1}}+\left(\beta y_{1}+y_{1} \varepsilon_{2} B\right) \frac{\partial}{\partial y_{1}}
$$

where $\varepsilon_{1}= \pm 1, \varepsilon_{2}= \pm 1$. We note that the signs of $\varepsilon_{1}$ and $\varepsilon_{2}$ are independent.

Having normalized $j^{3} X$ to 4.6 , next we consider the normalization of the homogenous terms of degree 5 . Let $X^{(5)}$ denote these terms, as obtained after the previous normalization step. We may write

$$
\begin{aligned}
X^{(5)} & =-x_{2}\left(a_{2,0} A^{2}+a_{1,1} A B+a_{0,2} B^{2}\right) \frac{\partial}{\partial x_{1}}+x_{1}\left(a_{2,0} A^{2}+a_{1,1} A B+a_{0,2} B^{2}\right) \frac{\partial}{\partial x_{2}} \\
& -y_{2}\left(b_{2,0} A^{2}+b_{1,1} A B+b_{0,2} B^{2}\right) \frac{\partial}{\partial y_{1}}+y_{1}\left(b_{2,0} A^{2}+b_{1,1} A B+b_{0,2} B^{2}\right) \frac{\partial}{\partial y_{1}}
\end{aligned}
$$

We now multiply the vector field $X$ by a function $h_{2}=1+\theta_{1} A^{2}+\theta_{2} A B+$ $\theta_{3} B^{2}$, where $\theta$ 's are parameters that will be specified further below. 
The third jet of the resulting vector field $h_{2} \cdot X$ is the same of $X$, and the terms of $h_{2} \cdot X$ of homogeneous degree 5 are

$$
\begin{aligned}
\tilde{X}^{(5)} & =-x_{2}\left(\left(a_{2,0}+\theta_{1}\right) A^{2}+\left(a_{1,1}+\theta_{2}\right) A B+\left(a_{0,2}+\theta_{3}\right) B^{2}\right) \frac{\partial}{\partial x_{1}} \\
& +x_{1}\left(\left(a_{2,0}+\theta_{1}\right) A^{2}+\left(a_{1,1}+\theta_{2}\right) A B+\left(a_{0,2}+\theta_{3}\right) B^{2}\right) \frac{\partial}{\partial x_{2}} \\
& -y_{2}\left(\left(b_{2,0}+\theta_{1}\right) A^{2}+\left(b_{1,1}+\theta_{2}\right) A B+\left(b_{0,2}+\theta_{3}\right) B^{2}\right) \frac{\partial}{\partial y_{1}} \\
& +y_{1}\left(\left(b_{2,0}+\theta_{1}\right) A^{2}+\left(b_{1,1}+\theta_{2}\right) A B+\left(b_{0,2}+\theta_{3}\right) B^{2}\right) \frac{\partial}{\partial y_{1}}
\end{aligned}
$$

We have to pass $\tilde{X}^{(5)}$ to

$$
X_{H}^{(5)}=-c x_{2} A^{2} \frac{\partial}{\partial x_{1}}-c x_{1} A^{2} \frac{\partial}{\partial x_{2}},
$$

so we we perform a change of coordinates generated by

$$
\begin{aligned}
& Y_{2}=x_{1}\left(\gamma_{1,0} A+\gamma_{0,1} B\right) \frac{\partial}{\partial x_{1}}+x_{2}\left(\gamma_{1,0} A+\gamma_{0,1} B\right) \frac{\partial}{\partial x_{2}} \\
& y_{1}\left(\delta_{1,0} A+\delta_{0,1} B\right) \frac{\partial}{\partial y_{1}}+y_{2}\left(\delta_{1,0} A+\delta_{0,1} B\right) \frac{\partial}{\partial y_{2}}
\end{aligned}
$$

where $\gamma$ 's and $\delta$ 's are real parameters. Now, just like in the previous case, we have to solve $\left[\tilde{X}^{(3)}, Y\right]=X^{(5)}-X_{H}^{(5)}$. One can check that a solution of this system is given by

$$
\begin{aligned}
\theta_{1} & =-b_{2,0} \\
\theta_{2} & =\frac{1}{6} \frac{-2 \gamma_{1,0} a_{1,0}+2 \gamma_{1,0} b_{1,0} \alpha-b_{2,0} \beta+a_{2,0} \beta}{\beta} \\
\theta_{3} & =-a_{0,2} \\
\delta_{1,0} & =-\frac{1}{2} \frac{\alpha\left(2 \gamma_{0,1} b_{1,0} \alpha+a_{1,1} \beta-2 \gamma_{0,1} a_{1,0} \beta-b_{1,1} \beta\right)}{\beta\left(b_{0,1} \alpha-a_{0,1} \beta\right)}, \\
\delta_{0,1} & =-\frac{1}{2} \frac{\alpha\left(-b_{0,2}+a_{0,2}\right)}{b_{0,1} \alpha-a_{0,1} \beta}
\end{aligned}
$$

with $\gamma_{1,0}, \gamma_{0,1}$ free variables.

Let us now fix the (Hamiltonian) polynomial vector field $j^{5} X$ as obtained by the above normalization procedure. We proceed to show that all higher order terms can be eliminated. In order to normalize $X^{(2 k+1)}, k \geq 3$, we first multiply the vector field by a function of the form

$$
h_{k}=1+\sum_{j=0}^{k} \theta_{k-j, j} A^{(k-j)} B^{j},
$$

with parameters $\theta_{k-j, j}$ and subsequently perform a change of coordinates, generated by a vector field of the form

$$
Y_{k}=\sum_{j=0}^{k-1} A^{k-1-j} B^{j}\left(\alpha_{k-1-j, j} z_{1} \frac{\partial}{\partial z_{1}}+\beta_{k-1-j, j} z_{2} \frac{\partial}{\partial z_{2}}\right)
$$


where $\alpha$ 's and $\beta$ 's are parameters. Then $\left(Y_{k}\right)_{*}\left(h_{k} X\right)$ takes the form

$$
\begin{aligned}
& i z_{1}\left(\begin{array}{l}
\left.1+\varepsilon_{1} A+c A^{2}+\sum_{j=0}^{k-1}\left(a_{k-j, j} \theta_{k-j, j}-2 \varepsilon_{1} \alpha_{k-1-j, j}\right) A^{k-j} B^{j}+\left(a_{0, k}+\theta_{0, k}\right) B^{k}\right) \frac{\partial}{\partial z_{1}} \\
+i z_{2}\left(\lambda+\varepsilon_{2} B+\sum_{j=1}^{k}\left(b_{k-j, j}+\lambda \theta_{k-j, j}-2 \varepsilon_{1} \beta_{k-1-j, j-1}\right) A^{k-j} B^{j}+\left(b_{k, 0}+\lambda \theta_{k, 0}\right) A^{k}\right) \frac{\partial}{\partial z_{2}}
\end{array} .\right.
\end{aligned}
$$

The elimination of all terms is equivalent to the solvability, with respect to the parameters $\theta_{i, j}, \alpha_{i, j}$ and $\beta_{i, j}$, of the following system of equations:

$$
\begin{aligned}
a_{k-j, j}+\theta_{k-j, j}-2 \varepsilon_{1} \alpha_{k-1-j, j} & =0, \quad j=0, \ldots, k-1 \\
b_{k-j, j}+\lambda \theta_{k-j, j}-2 \varepsilon_{2} \beta_{k-j, j-1} & =0, \quad j=1, \ldots, k, \\
a_{0, k}+\theta_{0, k} & =0 \\
b_{k, 0}+\lambda \theta_{k, 0} & =0
\end{aligned}
$$

where $a_{i, j}, b_{i, j} \in \mathbb{R}$ are constants whose values depend on the details of $X^{(2 k+1)}$. Due to the upper triangular form of this system of the equations, its solvability is evident.

We remark that we do not need the coefficients $\beta$ 's (in the same way we did not used the coefficients $\gamma$ 's in the normalization of 5-jet). The explanation for that is easy: while the reparametrization function $h_{k}$ eliminate the monomials in the first two coordinates, the change of coordinate eliminate the monomials in the last two equations.

\subsection{Non-semi-simple 1: 1 resonance: Theorem 3.3}

Without loss of generality, we assume that the eigenvalues of the vector fields are equal to $\pm i$. We start from the reversible Belitskii normal form (which has its nonlinear terms commuting with the transpose of the linear part)

$$
\begin{aligned}
X= & \left(-x_{2}+y_{1}-x_{2}\left(a_{1} A+a_{2} v\right)+\cdots\right) \frac{\partial}{\partial x_{1}}+\left(x_{1}+y_{2}+x_{1}\left(a_{1} A+a_{2} v\right)+\cdots\right) \frac{\partial}{\partial x_{2}} \\
& +\left(-y_{2}-y_{2}\left(a_{1} A+a_{2} v\right)+x_{1}\left(a_{3} v+a_{4} A\right)+\cdots\right) \frac{\partial}{\partial y_{1}} \\
& +\left(y_{1}+y_{1}\left(a_{1} A+a_{2} v\right)+x_{2}\left(a_{3} v+a_{4} A\right)+\cdots\right) \frac{\partial}{\partial y_{2}},
\end{aligned}
$$

where the dots denote the higher order terms and the $a$ 's denote parameters. The 3-jet of the vector field is Hamiltonian with respect to the standard symplectic form if and only if $a_{3}=-2 a_{1}$, with Hamiltonian function $H_{4}=\left(y_{1}^{2}+y_{2}^{2}\right) / 2+v+a_{1} A v+a_{2} v^{2} / 2-a_{4} A^{2} / 4$.

The condition $a_{3}=-2 a_{1}$ can be achieved by a re-scaling of time. After multiplication of $X$ by $f=1+\alpha A$ where $\alpha$ is a parameter, we get:

$$
\begin{aligned}
(f X)= & \left(-x_{2}+y_{1}-x_{2}\left(\left(a_{1}+\alpha\right) A+a_{2} v\right)+\underline{y_{1}(\alpha A)} \cdots\right) \frac{\partial}{\partial x_{1}} \\
& +\left(x_{1}+y_{2}+x_{1}\left(\left(a_{1}+\alpha\right) A+a_{2} v\right)+\underline{y_{2}(\alpha A)}+\cdots\right) \frac{\partial}{\partial x_{2}} \\
& +\left(-y_{2}-y_{2}\left(\left(a_{1}+\alpha\right) A+a_{2} v\right)+x_{1}\left(a_{3} v+a_{4} A\right)+\cdots\right) \frac{\partial}{\partial y_{1}} \\
& +\left(y_{1}+y_{1}\left(\left(a_{1}+\alpha\right) A+a_{2} v\right)+x_{2}\left(a_{3} v+a_{4} A\right)+\cdots\right) \frac{\partial}{\partial y_{2}}
\end{aligned}
$$


Using in turn another coordinate transformation to the Belitskii normal form, the underlined terms of 4.9 may be eliminated. Moreover, the remaining terms of 4.9 are not affected because they belong to the complement of the image of the homological operator; the change of coordinate

$$
I d+\left(\begin{array}{c}
x_{1}\left((1 / 2) \alpha \Delta_{2}\right)+y_{2}(-\alpha v) \\
x_{2}\left((1 / 2) \alpha \Delta_{2}\right)-y_{1}(-\alpha v) \\
0 \\
0
\end{array}\right)
$$

realize this. So the desired Hamiltonian form is obtained if $\alpha \in \mathbb{R}$ is chosen such that $a_{3}=-2\left(a_{1}+\alpha\right)$.

Starting with the $(2 k-3)$-jet in Hamiltonian form, with $k \geq 2$, we proceed to normalize the $(2 k-1)$-jet

$$
\begin{aligned}
X^{(2 k-1)}= & \left(-x_{2} \sum_{j=1}^{k} b_{j} A^{k-j} v^{j-1}\right) \frac{\partial}{\partial x_{1}}+\left(x_{1} \sum_{j=1}^{k} b_{j} A^{k-j} v^{j-1}\right) \frac{\partial}{\partial x_{2}}+ \\
& \left(-y_{2} \sum_{j=1}^{k} b_{j} A^{k-j} v^{j-1}+x_{1} \sum_{j=1}^{k} c_{j} A^{k-} v^{j-1}\right) \frac{\partial}{\partial y_{1}}+ \\
& \left(y_{1} \sum_{j=1}^{k} b_{j} A^{k-j} v^{j-1}+x_{2} \sum_{j=1}^{k} c_{j} A^{k-j} v^{j-1}\right) \frac{\partial}{\partial y_{2}}
\end{aligned}
$$

as follows. We first multiply by a function $f$ of the form $f=1+\sum_{j=1}^{k-1} \theta_{j} A^{k-j} v^{j-1}$ to obtain

$$
\begin{aligned}
& \tilde{X}^{(2 k-1)}=\left(-x_{2} \sum_{j=1}^{k}\left(b_{j}+\theta_{j}\right) A^{k-j} v^{j-1}+\underline{y_{1} \sum_{j=1}^{k-1} \theta_{j} A^{k-j} v^{j-1}}\right) \frac{\partial}{\partial x_{1}}+ \\
& \left(x_{1} \sum_{j=1}^{k}\left(b_{j}+\theta_{j}\right) A^{k-j} v^{j-1}+y_{2} \overline{\left.\sum_{j=1}^{k-1} \theta_{j} A^{k-j} v^{j-1}\right)} \frac{\partial}{\partial x_{2}}+\right. \\
& \left(-y_{2} \sum_{j=1}^{k}\left(b_{j}+\theta_{j}\right) A^{k-j} v^{j-1}+x_{1} \sum_{j=1}^{k} c_{j} A^{k-} v^{j-1}\right) \frac{\partial}{\partial y_{1}}+ \\
& \left(y_{1} \sum_{j=1}^{k}\left(b_{j}+\theta_{j}\right) A^{k-j} v^{j-1}+x_{2} \sum_{j=1}^{k} c_{j} A^{k-j} v^{j-1}\right) \frac{\partial}{\partial y_{2}}
\end{aligned}
$$

As above the underlined terms can be killed, without changing the other terms, by the change of coordinate

$$
I d+\left(\begin{array}{c}
x_{1} f(v, A, B)-y_{2} g(v, A) \\
x_{2} f(v, A, B)+y_{1} g(v, A) \\
0 \\
0
\end{array}\right),
$$

with $g(v, A)=\sum_{j=0}^{k-2} \theta_{k-j-1} v^{k-j-1} A^{j}$ and $f(v, A, B)=B \sum_{j=0}^{k-2} \theta_{1+j} A^{k-j} v^{j}$.

So and we may choose $\theta_{j}, j=1, \ldots, k$ such that

$$
j c_{j+1}=-2(k-j)\left(b_{j}+\theta_{j}\right), \quad j=1, \ldots, k .
$$

\section{3. $1: 2$ resonance: Theorem 3.5}

The aim is to prove that in the case of $1: 2$ resonance, generically the vector field is formally orbitally equivalent to a Hamiltonian vector field. 
We start with a reversible vector field $X$ with linear part having eigenvalues $( \pm \lambda i, \pm 2 \lambda i) . \varphi$ time reversible, where $\varphi\left(z_{1}, z_{2}\right)=-\left(\bar{z}_{1}, \bar{z}_{2}\right)$. Therefore $X$ can be assumed to be in normal form (3.5), where, due to the reversibility, the coefficients of all odd degree homogenous terms of $X$ (in terms of $z_{1}, \bar{z}_{1}, z_{2}, \bar{z}_{2}$ ) are purely imaginary and the coefficients of all even degree homogenous terms are real.

We first observe some useful equalities between the variables:

$$
\begin{aligned}
& C D=A^{2} B, \quad \bar{z}_{1} z_{2} A^{k}=z_{1} A^{k-1} C, \quad z_{1}^{2} D^{k}=z_{2} A^{2} D^{k-1}, \\
& \bar{z}_{1} z_{2} C^{k}=z_{1} A B C^{k-1}, \quad z_{1}^{2} B^{k}=z_{2} B^{k-1} C
\end{aligned}
$$

where $k$ is any positive integer.

Up to linear scalings of $z_{1}$ and $z_{2}$, the genericity conditions $(3.6)$ can be divided into two cases: (i) $f_{2}(0,0)=-2, g_{2}(0,0)=1$; and (ii) $f_{2}(0,0)=2$, $g_{2}(0,0)=1$. Below we only consider the first case, i.e., $f_{2}(0,0)=-2$ and $g_{2}(0,0)=1$. The other case can be treated exactly in the same way.

We denote by $X^{(n)}=P_{n} \partial / \partial z_{1}+Q_{n} \partial / \partial z_{2}$, where $P_{n}$ and $Q_{n}$ are homogenous polynomial of degree $n$ of their variables. For simplicity, we often use the tuple $\left(P_{n}, Q_{n}\right)$ to denote the above named $X^{(n)}$. We may write:

$$
\begin{aligned}
& X^{(2)}=\left(-2 \bar{z}_{1} z_{2}, z_{1}^{2}\right) \\
& X^{(3)}=\left(i z_{1}\left(a_{2} A+a_{3} B\right), i z_{2}\left(b_{2} A+b_{3} B\right)\right) \\
& X^{(4)}=\left(z_{1}\left(a_{4} C+a_{5} D\right)+a_{6} \bar{z}_{1} z_{2} B, z_{2}\left(b_{4} C+b_{5} D\right)+b_{6} z_{1}^{2} A\right), \ldots
\end{aligned}
$$

To express $X^{(n)}$ in an explicit way for general $n$, one needs to distinguish the following cases $X^{(6 k+j)}$, for $j=1,2, \ldots, 6$. This is because that $A$ and $B$ are terms of degree 2 while $C$ and $D$ are terms of degree 3 .

Let

$$
X^{(6 k+2 n)}=\left(I_{n}, I I_{n}\right), \quad n=0,1,2
$$

with

$$
\begin{aligned}
I_{n}= & z_{1} \sum_{s=\sigma_{n}}^{k} \sum_{j=0}^{3 s+n-2}\left(f_{s j} C^{2 k-2 s+1}+g_{s j} D^{2 k-2 s+1}\right) A^{3 s+n-2-j} B^{j} \\
& +z_{1} z_{2} \sum_{l=0}^{\omega_{n}} h_{l} D^{2 l} B^{3 k-3 l+n-1} \\
I I_{n}= & z_{2} \sum_{s=\sigma_{n}}^{k} \sum_{j=0}^{3 s+n-2}\left(\tilde{f}_{s j} C^{2 k-2 s+1}+\tilde{g}_{s j} D^{2 k-2 s+1}\right) A^{3 s+n-2-j} B^{j} \\
& +z_{1}^{2} \sum_{l=0}^{\omega_{n}} \tilde{h}_{l} C^{2 l} B^{3 k-3 l+n-1}
\end{aligned}
$$

and

$$
X^{(6 k+2 n+1)}=\left(I^{n}, I I^{n}\right), \quad n=0,1,2
$$


where

$$
\begin{aligned}
I^{n}= & i z_{1} \sum_{j=0}^{3 k+n} a_{j} A^{3 k+n-j} B^{j}+i z_{1} \sum_{s=0}^{k-1} \sum_{j=0}^{3 s+n}\left(f_{s j} C^{2 k-2 s}+g_{s j} D^{2 k-2 s}\right) A^{3 s+n-j} B^{j} \\
& +i \bar{z}_{1} z_{2} \sum_{l=0}^{\nu_{n}} h_{l} D^{2 l+1} B^{3 k-3 l+n-2} \\
I I^{n}= & i z_{2} \sum_{j=0}^{3 k+n} b_{j} A^{3 k+n-j} B^{j}+i z_{2} \sum_{s=0}^{k-1} \sum_{j=0}^{3 s+n}\left(\tilde{f}_{s j} C^{2 k-2 s}+\tilde{g}_{s j} D^{2 k-2 s}\right) A^{3 s+n-j} B^{j} \\
& +i z_{1}^{2} \sum_{l=0}^{\nu_{n}} \tilde{h}_{l} C^{2 l+1} B^{3 k-3 l+n-2}
\end{aligned}
$$

with $\left.\sigma_{n}:=\mid\right] \frac{2-n}{3}\left[\mid, \nu_{n}:=\llbracket k+\frac{n-2}{3} \|\right.$ and $\left.\omega_{n}:=\llbracket \mid k+\frac{n-1}{3} \|\right]$ where $[|\alpha|]$ is the biggest integer smaller than $\alpha$ and $\mid] \alpha[\mid$ is the smallest integer greater than $\alpha$

Moreover, a straightforward calculation gives us the number of resonant terms in $X^{(n)}$ :

$$
\begin{aligned}
& X^{(6 k)} \text { contains } 6 k^{2}+4 k \text { resonant terms; } \\
& X^{(6 k+1)} \text { contains } 6 k^{2}+6 k+2 \text { resonant terms; } \\
& X^{(6 k+2)} \text { contains } 6 k^{2}+8 k+2 \text { resonant terms; } \\
& X^{(6 k+3)} \text { contains } 6 k^{2}+10 k+4 \text { resonant terms; } \\
& X^{(6 k+4)} \text { contains } 6 k^{2}+12 k+6 \text { resonant terms; } \\
& X^{(6 k+5)} \text { contains } 6 k^{2}+14 k+8 \text { resonant terms. }
\end{aligned}
$$

It is clear that in the above expressions, we have taken in account the relations 4.10. Observe that when we say numbers of resonant terms we mean the numbers of mutually different resonant terms.

Note that $X^{(6 k+j+1)}$ has $2 k+2$ more resonant terms than $X^{(6 k+j)}$ for $j=$ $0,2,3,4,5$, whereas $X^{(6 k+2)}$ has $2 k$ more terms than $X^{(6 k+1)}$

Here we have to prove that for each $k \geq 2$ and assuming the generic condition (3.6) $-1, X^{(k)}$ can be normalized in the following way

$$
X^{(k)}=i \frac{\partial H^{(k+1)}}{\partial \bar{z}_{1}} \frac{\partial}{\partial z_{1}}+i \frac{\partial H^{(k+1)}}{\partial \bar{z}_{2}} \frac{\partial}{\partial z_{2}}
$$

where $H^{(k+1)}$ is a function of homogenous degree $k+1$ of $z_{1}, \bar{z}_{1}$ and $z_{2}, \bar{z}_{2}$.

The validity of the 4.15 for $X^{(1)}$ is trivial, i.e. $X^{(1)}=i\left(\partial H^{(2)} / \partial \bar{z}_{1}\right.$, $\left.\partial H^{(2)} / \partial \bar{z}_{2}\right)$, where $H^{(2)}=\lambda(A+2 B)$. It is also valid for $X^{(2)}$. In fact, since $X^{(2)}=\left(-2 \bar{z}_{1} z_{2}, z_{1}^{2}\right)$, one can take $H^{(3)}=i(C-D)$ so that $X^{(2)}=i\left(\partial H^{(3)} / \partial \bar{z}_{1}\right.$, $\left.\partial H^{(3)} / \partial \bar{z}_{2}\right)$. Note that $H^{(3)}$ contains just real terms, since $C-D$ is purely imaginary. Starting from $X^{(3)}$, below we show that the statement is also true, without any condition imposed on the higher order nonlinear terms. 
To see the validity of 4.15 for $X^{(3)}$, we assume that $X^{(3)}=\left(i z_{1}\left(a_{1} A+\right.\right.$ $\left.\left.a_{2} B\right), i z_{2}\left(b_{1} A+b_{2} B\right)\right)$, where $a_{1,2}$ and $b_{1,2}$ are real. We need to show that $X^{(3)}$ can be transformed to $\tilde{X}^{(3)}$, where the corresponding coefficients $\tilde{a}_{1}, \tilde{a}_{2}, \tilde{b}_{1}$ and $\tilde{b}_{2}$ satisfy the equality $\tilde{a}_{2}=\tilde{b}_{1}$. Indeed, if so, then we have $\tilde{X}^{(3)}=i\left(\partial H^{(4)} / \partial \bar{z}_{1}\right.$, $\left.\partial H^{(4)} / \partial \bar{z}_{2}\right)$, where $H^{(4)}=\frac{\tilde{a}_{1}}{2} A^{2}+\tilde{a}_{2} A B+\frac{\tilde{b}_{2}}{2} B^{2}$.

To get the equality, we perform a resonant change of coordinates $\left(z_{1}, z_{2}\right) \rightarrow$ $I d+\left(\alpha \bar{z}_{1} z_{2}, \beta z_{1}^{2}\right)$ with inverse $\left(z_{1}, z_{2}\right) \rightarrow I d-\left(\alpha \bar{z}_{1} z_{2}, \beta z_{1}^{2}\right)+\left(\alpha \beta z_{1} A+\alpha^{2} z_{1} B, 2 \alpha \beta z_{2} A\right)+$ $o(4)$, which obviously keeps $X^{(2)}$ unchanged and brings the following contribution to the original $X^{(3)}:\left(-(\alpha+2 \beta) z_{1} A-4 \alpha z_{1} B, 2(\alpha+4 \beta) z_{2} A\right)$. In other words,

$$
\tilde{a}_{1}=a_{1}-(\alpha+2 \beta), \quad \tilde{a}_{2}=a_{2}-4 \alpha, \quad \tilde{b}_{1}=b_{1}+2(\alpha+4 \beta), \quad \tilde{b}_{2}=b_{2} .
$$

Thus it is always possible to choose suitable $\alpha$ (or $\beta$ ) to meet the requirement.

We emphasize that there are now six cases $X^{(6 k+j)}, j=1,2, \ldots, 6$ to be analyzed. We divide our analysis in two cases: i) the polynomial that contains just even order terms, $X^{(6 k+2 n)}$ and otherwise ii) $X^{(6 k+2 n+1)}$.

Consider $X^{(6 k+2 n)}$ written in the general form 4.11). Next step is to show that such terms can be normalized in such a way that its first component $\left(\dot{z}_{1},\right)$ takes the form

$$
\begin{aligned}
\tilde{I}_{n}= & z_{1} \sum_{j=0}^{3 k+n-2}\left[\alpha_{j}(3 k+n-1-j) C-\alpha_{j}(3 k+n+1-j) D\right] A^{3 k+n-2-j} B^{j} \\
& +2 \bar{z}_{1} z_{2} \alpha_{3 k+n-1} B^{3 k+n-1}
\end{aligned}
$$

and the second, corresponding to $\dot{z}_{2}$, is

$$
\begin{aligned}
\tilde{I I}_{n}= & z_{2} \sum_{j=0}^{3 k+n-2}\left[\alpha_{j+1}(j+2) C+\alpha_{j+1}(J+1) D\right] A^{3 k+n-1-j} B^{j} \\
& +z_{1}^{2} \alpha_{o} A^{3 k+n-1}
\end{aligned}
$$

If such normalization can be realized then we can take the following function $H^{(6 k+2 n+1)}$ as the generating function of the normalized $X^{(6 k+2 n)}$.

$$
H^{(6 k+2 n+1)}=-i \sum_{j=0}^{3 k+n-1} \alpha_{j}(C-D) A^{3 k+n-1-j} B^{j},
$$

since one can check directly that $X^{(6 k+2 n)}=\left(i \partial H / \partial \bar{z}_{1}, i \partial H / \partial \bar{z}_{2}\right)$.

Similar argument can be used for the second case $X^{(6 k+2 n+1)}$. In this case all terms in $C$ and $D$ can be killed. Hence $H^{(6 k+2 n+2)}$ can be taken having the form $H(A, B)$.

We back now to the case $6 k+2 n$ : first of all we show that the independent terms of $C$ and $D$ with exponents greater than 1 can be killed. After that we show that the remaining coefficients in (4.12), denoted by $f_{j}:=f_{k j}, g_{j}:=$ $g_{k j}, \quad \tilde{f}_{j}:=\tilde{f}_{k j}, \quad \tilde{g}_{j}:=\tilde{g}_{k j}, j=0, \ldots, 3 k+n-3, h_{o}$ e $\tilde{h}_{o}$, can be selected to satisfy the following compatibility conditions: 


$$
\begin{array}{rr}
(j+2) f_{j+1}=(3 k+n-2-j) \tilde{f}_{j} & \\
(j+1) g_{j+1}=(3 k+n-j) \tilde{g}_{j} & , j=0, \ldots, 3 k+n-3 \\
f_{o}=(3 k+n-1) \tilde{h}_{o} & \\
2 \tilde{g}_{3 k+n-2}=(3 k+n-1) h_{o} & \\
(3 k+n+1-j) f_{j}=-(3 k+n-1-j) g_{j} & \\
(j+1) \tilde{f}_{j}=-(j+2) \tilde{g}_{j} & , j=0, \ldots, 3 k+n-2 .
\end{array}
$$

To do that we consider a coordinate system given by $\left(z_{1}, z_{2}\right) \mapsto\left(z_{1}, z_{2}\right)+$ $Y^{(6 k+2 n-1)}\left(z_{1}, z_{2}\right)$ with

$$
Y^{(6 k+2 n-1)}\left(z_{1}, z_{2}\right)=\left(F\left(z_{1}, \bar{z}_{1}, z_{2}, \bar{z}_{2}\right), G\left(z_{1}, \bar{z}_{1}, z_{2}, \bar{z}_{2}\right)\right),
$$

where

$$
\begin{aligned}
F & =z_{1} \sum_{j=0}^{3 k+n-1} \varepsilon_{j} A^{3 k+n-1-j} B^{j} \\
& +z_{1} \sum_{s=\tilde{\sigma}_{n}}^{k-1} \sum_{j=0}^{3 s+n-1}\left(\alpha_{s j} C^{2 k-2 s}+\beta_{s j} D^{2 k-2 s}\right) A^{3 s+n-1-j} B^{j} \\
& +\bar{z}_{1} z_{2} \sum_{l=0}^{\eta_{n}} \gamma_{l} D^{2 l+1} B^{3 k-3 l+n-3},
\end{aligned}
$$

and

$$
\begin{aligned}
G & =z_{2} \sum_{j=0}^{3 k+n-1} \tilde{\varepsilon}_{j} A^{3 k+n-1-j} B^{j} \\
& +z_{2} \sum_{s=1}^{k-1} \sum_{j=0}^{3 s+n-1}\left(\tilde{\alpha}_{s j} C^{2 k-2 s}+\tilde{\beta}_{s j} D^{2 k-2 s}\right) A^{3 s+n-1-j} B^{j} \\
& +z_{1}^{2} \sum_{l=0}^{\eta_{n}} \tilde{\gamma}_{l} C^{2 l+1} A^{3 k-3 l+n-3} .
\end{aligned}
$$

with $\left.\tilde{\sigma}_{n}:=\mid\right] \frac{1-n}{3}\left[\mid\right.$ and $\left.\eta_{n}:=\llbracket\left|k+\frac{n-3}{3}\right|\right]$

By doing so we can decouple the system of homological equations in the following way. We first perform changes of coordinates to eliminate the resonant terms corresponding to terms containing $C$ and $D$ with exponent bigger than 1. Then we prove that there exists a suitable function of the form $f=1+$ $\sum_{j=0}^{3 k+n-2}\left[\theta_{j} C+\psi_{j} D\right] A^{3 k+n-2-j} B^{j}$, such that under the multiplication by $f$ the compatible conditions can be satisfied. Note that the function $f$ does not create any new resonant terms containing $C$ and $D$ in $X^{(6 k+2 n)}$. When these two steps are satisfied the system $X^{(6 k+2 n)}$ gets the desired normalization.

Recall that $X^{(2)}=\left(-2 \bar{z}_{1} z_{2}, z_{1}^{2}\right)$ and $Y^{(6 k+2 n-1)}$ is given by 4.20$)$. The first step of its normalization is based on the Lie bracket $\left[X^{(2)}, Y^{(6 k+2 n-1)}\right]$. A straightforward calculation yields that

$$
\left[X^{(2)}, Y^{(6 k+2 n-1)}\right]=I \frac{\partial}{\partial z_{1}}+J \frac{\partial}{\partial z_{2}},
$$

where $I=I_{1}+I_{2}$, and $J=J_{1}+J_{2}$ with 


$$
\begin{aligned}
I_{1}= & \mathcal{O}\left(C^{1}\right)+z_{1} \sum_{s=\tilde{\sigma}_{n}+1}^{k-1} C^{2 k-2 s+1}\left\{\left(\alpha_{s, 1}+2 \tilde{\gamma}_{k-s}\right) A^{3 s+n-2}+\right. \\
& +\sum_{j=1}^{3 s+n-3}\left[\alpha_{s-1, j-1}(4 k-s+n+2-j)+2 \tilde{\alpha}_{s-1, j-1}+\right. \\
& \left.\left.+(j+1) \alpha_{s, j+1}\right] A^{3 s+n-2-j} B^{j}+(3 s+n-1) \alpha_{s, 3 s+n-1} B^{3 s+n-2}\right\}+ \\
& +z_{1} C^{2 k-2 \tilde{\sigma}_{n}+1}\left(\mathcal{X}_{0}(n)\left[2 \tilde{\gamma}_{k-1} A+\sum_{j=0}^{1}(j+1) \alpha_{1, j+1} A^{1-j} B^{j}\right]+\mathcal{X}_{2}(n) \alpha_{0,1}\right) \\
& +\mathcal{O}\left(D^{1}\right)+ \\
& z_{1} \sum_{\tilde{\sigma}_{n}+1}^{k-1} D^{2 k-2 s+1}\left\{\sum _ { j = 0 } ^ { 3 s + n - 4 } \left[-2 \beta_{s j}(3 s+n-j)++\beta_{s-1, j}(2 k-2 s+2+j)+\right.\right. \\
& \left.+2 \tilde{\beta}_{s j}\right] A^{3 s+n-2-j} B^{j}+ \\
& +\left[-6 \beta_{s, 3 s+n-3}+\tilde{\beta}_{s, 3 s+n-3}+\gamma_{k-s}(2 k+s+n-1)\right] A B^{3 s+n-2}+ \\
& \left.+\left[-4 \beta_{s, 3 s+n-2}+2 \tilde{\beta}_{s, 3 s+n-2}\right] B^{3 s+n-2}\right\}+ \\
& +z_{1} D^{2 k-2 \tilde{\sigma}_{n}+1}\left(\mathcal{X}_{0}(n)\left[2 k \gamma_{k-1} A+\sum_{j=0}^{1}\left[-2 \beta_{1, j}(3-j)+2 \tilde{\beta}_{1, j}\right] A^{1-j} B^{j}\right]+\right. \\
& \left.+\mathcal{X}_{2}(n)\left[-4 \beta_{00}+2 \tilde{\beta}_{00}\right]\right)
\end{aligned}
$$

and

$$
\begin{aligned}
I_{2} & =\bar{z}_{1} z_{2}\left(-2 \varepsilon_{3 k+n-1}+2 \tilde{\varepsilon}_{3 k+n-1}\right) B^{3 k+n-1}+ \\
& +\bar{z}_{1} z_{2} \sum_{s=\tilde{\sigma}_{n}}^{k-1}\left(-2 \beta_{s, 3 s+n-1}+2 \tilde{\beta}_{s, 3 s+n-1}\right) D^{2 k-2 s} B^{3 s+n-1}
\end{aligned}
$$


In a similar way, we write $J=J_{1}+J_{2}$ where

$$
\begin{aligned}
J_{1}= & \mathcal{O}\left(C^{1}\right)+z_{2} \sum_{s=\tilde{\sigma}_{n}+1}^{k-1} C^{2 k-2 s+1}\left\{\left[2 \tilde{\alpha}_{s, 1}-2 \alpha_{s, 1}-2 \tilde{\gamma}_{k-s}(4 k-s+n-1)\right] A^{3 s+n-2}+\right. \\
& +\sum_{j=1}^{3 s+n-3}\left[-2(4 k-s+n+1-j) \tilde{\alpha}_{s-1, j-1}+(j+2) \tilde{\alpha}_{s, j+1}-\right. \\
& \left.\left.-2 \alpha_{s, j+1}\right] A^{3 s+n-2-j} B^{j}+\left[(3 s+n) \tilde{\alpha}_{s, 3 s+n-1}-2 \alpha_{s, 3 s+n-1}\right] B^{3 s+n-2}\right\}+ \\
& +z_{2} C^{2 k-2 \tilde{\sigma}_{n}+1}\left(\mathcal{X}_{0}(n)\left[-8 \tilde{\gamma}_{k-1} A+\sum_{j=0}^{1}\left[(j+2) \tilde{\alpha}_{1, j+1}-2 \alpha_{1, j+1}\right] A^{1-j} B^{j}\right]\right. \\
& \left.+\mathcal{X}_{2}(n)\left[2 \tilde{\alpha}_{0,1}-2 \alpha_{0,1}\right]\right)+\mathcal{O}\left(D^{1}\right)+ \\
& +z_{2} \sum_{s=\tilde{\sigma}_{n}+1}^{k-1} D^{2 k-2 s+1}\left\{\sum _ { j = 0 } ^ { 3 s + n - 4 } \left[-2(3 s+n-1-j) \tilde{\beta}_{s j}+\right.\right. \\
& \left.+(3 k-2 s+3+j) \tilde{\beta}_{s-1, j}-2 \beta_{s-1, j}\right] A^{3 s+n-2-j} B^{j} \\
& \left.-\left[4 \tilde{\beta}_{s, 3 s+n-3}+2 \gamma_{k-s}\right] A B^{3 s+n-3}-2 \tilde{\beta}_{s, 3 s+n-2} B^{3 s+n-2}\right\}+ \\
& +z_{2} D^{2 k-2 \tilde{\sigma}_{n}+1}\left(\mathcal{X}_{0}(n)\left[-2 \gamma_{k-1} A+\sum_{j=0}^{1}-2(3-1-j) \tilde{\beta}_{1, j} A^{1-j} B^{j}\right]\right. \\
& \left.+\mathcal{X}_{2}(n)\left(-2 \tilde{\beta}_{0,0}\right)\right)
\end{aligned}
$$

and

$$
\begin{aligned}
J_{2} & =z_{1}^{2}\left(\tilde{\varepsilon}_{0}-2 \varepsilon_{0}\right) A^{3 k+n-1}+ \\
& +z_{1}^{2} \sum_{s=\tilde{\sigma}_{n}}^{k-1}\left(\tilde{\alpha}_{s, 0}-2 \alpha_{s, 0}\right) C^{2 k-2 s} A^{3 s+n-1}
\end{aligned}
$$

where $\mathcal{X}_{R}$ is the characteristic function of the set $R$.

The elimination of the terms having exponents greater than 1 in $C$ and $D$ in the normal form is equivalent to get the solubility of the following systems: 


$$
\begin{aligned}
& \left\{\begin{array}{l}
\alpha_{s, 1}+2 \tilde{\gamma}_{k-s}=\epsilon_{6 k+2 n-1}^{(1)} \\
(4 k-s+n+2-j) \alpha_{s-1, j-1}+\tilde{\alpha}_{s-1, j-1}+(j+1) \alpha_{s, j+1}=\epsilon_{6 k+2 n-1}^{(2)} \\
(3 s+n-1) \alpha_{s, 3 s+n-1}=\epsilon_{6 k+2 n-1}^{(3)}
\end{array}\right. \\
& \mathcal{X}_{0}(n)\left(2 \tilde{\gamma}_{k-1}+\alpha_{1,1}\right)=\mathcal{X}_{0}(n) \epsilon_{6 k+2 n-1}^{(4)} \\
& \mathcal{X}_{0}(n) \alpha_{1,2}=\mathcal{X}_{0}(n) \epsilon_{6 k+2 n-1}^{(5)} \\
& \mathcal{X}_{2}(n) \alpha_{0,1}=\mathcal{X}_{2}(n) \epsilon_{6 k+2 n-1}^{(6)} \\
& \left\{2 \tilde{\alpha}_{s, 1}-2 \alpha_{s, 1}-(4 k-s+n-1) \tilde{\gamma}_{k-s}=\epsilon_{6 k+2 n-1}^{(7)}\right. \\
& -2(4 k-s+n+1-j) \tilde{\alpha}_{s-1, j-1}+(j+2) \tilde{\alpha}_{s, j+1}-2 \alpha_{s, j+1}=\epsilon_{6 k+2 n-1}^{(8)} \\
& (3 s+n) \tilde{\alpha}_{s, 3 s+n-1}-2 \alpha_{s, 3 s+n-1}=\epsilon_{6 k+2 n-1}^{(9)} \\
& \mathcal{X}_{0}(n)\left(-8 k \tilde{\gamma}_{k-1}+2 \tilde{\alpha}_{1,1}-2 \alpha_{1,1}\right)=\mathcal{X}_{0}(n) \epsilon_{6 k+2 n-1}^{(10)} \\
& \mathcal{X}_{0}(n)\left(3 \tilde{\alpha}_{1,2}-2 \alpha_{1,2}\right)=\mathcal{X}_{0}(n) \epsilon_{6 k+2 n-1}^{(11)} \\
& \mathcal{X}_{2}(n)\left(2 \tilde{\alpha}_{0,1}-2 \alpha_{0,1}\right)=\mathcal{X}_{2}(n) \epsilon_{6 k+2 n-1}^{(12)} \\
& \left(\tilde{\alpha}_{s, 0}-2 \alpha_{s, 0}=\epsilon_{6 k+2 n-1}^{(12+s)}, \quad s=\tilde{\sigma}_{n}, \ldots, k-1\right.
\end{aligned}
$$

where $\epsilon_{6 k+2 n-1}^{(j)}$ are some constants, $s=\tilde{\sigma}_{n}+1, \ldots, k-1, j=1, \ldots, 3 s+n-3$ (except in the last equation) and 


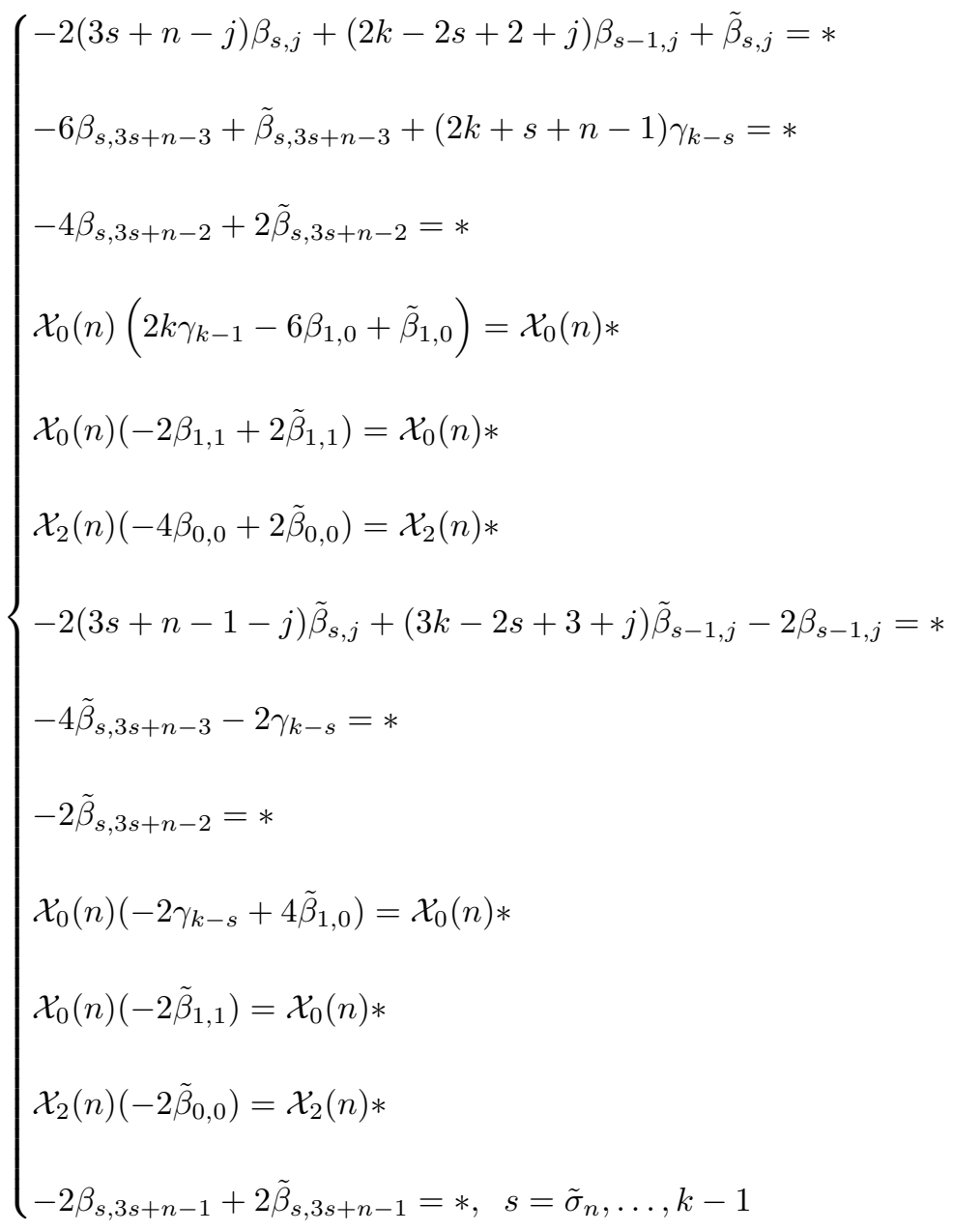

with $s=\tilde{\sigma}_{n}+1, \ldots, k-1$ and $j=0, \ldots, 3 s+n-4$ (except in the last equation).

We analyze now the system 4.22):

Step 1: From the equations 3 and 9 in 4.22 we find the values $\alpha_{s, 3 s+n-1}$ and $\tilde{\alpha}_{s, 3 s+n-1}, s=\tilde{\sigma}_{n}+1, \ldots, k-1$.

Step 2: From the equations 1 and 7 in 4.22 we get:

$$
\begin{aligned}
& 2 \tilde{\gamma}_{k-s}=*-\alpha_{s, 1} \\
& 2 \tilde{\alpha}_{s, 1}=(-4 k+s-n+3) \alpha_{s, 1}+*
\end{aligned}
$$

and so once $\alpha_{s, 1}$ for $s=\tilde{\sigma}_{n}+1, \ldots, k-1$ is chosen we get the values of $\tilde{\alpha}_{s, 1}$ and $\tilde{\gamma}_{k-s}, s=\tilde{\sigma}_{n}+1, \ldots, k-1$. 
Step 3: From the equations 2 and 8 in 4.22 we get:

$$
\begin{aligned}
(j+1) \alpha_{s, j+1}= & *-2 \tilde{\alpha}_{s-1, j-1}-(4 k-s+n+2-j) \alpha_{s-1, j-1} \\
(j+2) \tilde{\alpha}=\quad & *+2(4 k-s+n+1-j) \tilde{\alpha}_{s-1, j-1}-\frac{4}{j+1} \tilde{\alpha}_{s-1, j-1} \\
& -\frac{2(4 k-s+n+2-j)}{j+1} \alpha_{s-1, j-1}
\end{aligned}
$$

with $s=\tilde{\sigma}_{n}+1, \ldots, k-1$ e $j=1, \ldots, 3 s+n-3$.

In this way once $\tilde{\alpha}_{s-1, j-1}$ and $\alpha_{s-1, j-1}$ are chosen we may recursively find the values of $\alpha_{s, j+1}$ and $\tilde{\alpha}_{s, j+1}$ for $s=\tilde{\sigma}_{n}+1, \ldots, k-1$ and $j=1, \ldots, 3 s+n-3$.

Observe that the later procedure does not imply in any overlapping with step 2 and step 1 since the relations $j+1 \geq 2$ and $j \leq 3 s+n-3$ are satisfied respectively.

Step 4: The fixed parameters in the preceding steps are not an obstruction to solve the equation 13 in 4.22 since that now we have $j=0$.

Step 5: When $n=0$ there are four extra equations given by equations $4,5,10$ and 11 in 4.22. In this way such equations determine the values $\alpha_{1,2}$ and $\tilde{\alpha}_{1,2}$. However there is no overlapping with the step 3 since in that case we have $s \geq \tilde{\sigma}_{0}+1=2$. Moreover the equations 4 and 10 in 4.22 has solution since $\gamma_{k-s}$ and $\alpha_{1,1}$ are not fixed.

Step 6: When $n=2$ two extra equations are solved by fixing $\alpha_{0,1}$ e $\tilde{\alpha}_{0,1}$.

The system 4.23 is solved in a similar way as 4.22).

Hence the following normal form is achieved:

$$
\begin{aligned}
\tilde{X}^{(6 k+2 n)} & =\left(z_{1} \sum_{j=0}^{3 k+n-2}\left(f_{j} C+g_{j} D\right) A^{3 k+n-2-j} B^{j}+\bar{z}_{1} z_{2} h_{o} B^{3 k+n-1}\right) \frac{\partial}{\partial z_{1}} \\
& +\left(z_{2} \sum_{j=0}^{3 k+n-2}\left(\tilde{f}_{j} C+\tilde{g}_{j} D\right) A^{3 k+n-2-j} B^{j}+z_{1}^{2} \tilde{h}_{o} A^{3 k+n-1}\right) \frac{\partial}{\partial z_{2}}
\end{aligned}
$$

The goal now is to put the system (4.24) in the Hamiltonian form (see 4.18). So 4.24 has to satisfy the compatibility conditions 4.19).

We proceed now by multiplying the system $\tilde{X}^{(6 k+2 n)}$ by a function having the form

$$
f=1+\sum_{j=0}^{3 k+n-2}\left(\theta_{j} C+\psi_{j} D\right) A^{3 k+n-2-j} B^{j} .
$$

We get then a new system $\tilde{X}^{(6 k+2 n)}$ expressed by:

$$
\begin{aligned}
& \left(z_{1} \sum_{j=0}^{3 k+n-2}\left(\left(f_{j}+\lambda \theta_{j}\right) C+\left(g_{j}+\lambda \psi_{j}\right) D\right) A^{3 k+n-2-j} B^{j}+\bar{z}_{1} z_{2} h_{o} B^{3 k+n-1}\right) \frac{\partial}{\partial z_{1}}+ \\
& +\left(z_{2} \sum_{j=0}^{3 k+n-2}\left(\left(\tilde{f}_{j}+2 \lambda \theta_{j}\right) C+\left(\tilde{g}_{j}+2 \lambda \psi_{j}\right) D\right) A^{3 k+n-2-j} B^{j}+z_{1}^{2} \tilde{h}_{o} A^{3 k+n-1}\right) \frac{\partial}{\partial z_{2}}
\end{aligned}
$$


The compatibility conditions are now expressed by:

$$
\begin{array}{r}
(j+2)\left[f_{j+1}+\lambda \theta_{j+1}\right]=(3 k+n-2-j)\left[\tilde{f}_{j}+2 \lambda \theta_{j}\right] \\
(j+1)\left[g_{j+1}+\lambda \psi_{j+1}\right]=(3 k+n-j)\left[\tilde{g}_{j}+2 \lambda \psi_{j}\right] \\
(3 k+n+1-j)\left[j_{j}+\lambda \theta_{j}\right]=-(3 k+n-1-j)\left[g_{j}+\lambda \psi_{j}\right] \\
(j+1)\left[\tilde{f}_{j}+2 \lambda \theta_{j}\right]=-(j+2)\left[\tilde{g}_{j}+2 \lambda \psi_{j}\right] \\
f_{o}+\lambda \theta_{o}=(3 k+n-1) \tilde{h}_{o} \\
2\left[\tilde{g}_{3 k+n-2}+2 \lambda \psi_{3 k+n-2}\right]=(3 k+n-1) h_{o}
\end{array}
$$

where $j=0, \ldots, 3 k+n-3$ in the first two equations and $j=0, \ldots, 3 k+n-2$ in the last two equations. Remark that the solutions of the two last equations can be obtained independently of the values of $f_{o}, \theta_{o}, \tilde{g}_{3 k+n-2}$ e $\psi_{3 k+n-2}$. In fact, in 4.20 we have:

$$
\begin{aligned}
& I_{2}=\bar{z}_{1} z_{2}\left(-2 \varepsilon_{3 k+n-1}+2 \tilde{\varepsilon}_{3 k+n-1}\right) B^{3 k+n-1}+\cdots \\
& J_{2}=z_{1}^{2}\left(\tilde{\varepsilon}_{o}-2 \varepsilon_{o}\right) A^{3 k+n-1}+\cdots
\end{aligned} .
$$

So we may collect $\varepsilon_{o}, \tilde{\varepsilon}_{o}, \varepsilon_{3 k+n-1}$ and $\tilde{\varepsilon}_{3 k+n-1}$ in such a way that all the required conditions on the last two equations are fulfilled. So we find no incompatibility between the systems 4.22 and 4.23 . Therefore our concerning now is to analize the other equations (namely $1,2,3$ and 4) in 4.25. We argue by recursion and we assume that such equations are solved for all $k=2,3, . ., j$. One sees that equations 3 and 4 for $j+1$ are naturally satisfied with respect to $\theta_{j+1}$ and $\psi_{j+1}$ specified by the equations 1 and 2 in the last step. Next we solve 3 and 4 for $\theta_{o}$ and $\psi_{o}$. Collecting $\theta_{j+1}$ and $\psi_{j+1}, j=0, \ldots, 3 k+n-3$ satisfying equations 1 and 2 in 4.25 we derive that the jet $X^{(6 k+2 n)}$ takes the form (4.18).

Consider now the case given by $X^{6 k+2 n+1}$ :

First of all, consider a coordinate system given by $\left(z_{1}, z_{2}\right) \mapsto\left(z_{1}, z_{2}\right)+$ $Y^{(6 k+2 n)}\left(z_{1}, z_{2}\right)$ with

$$
Y^{(6 k+2 n)}\left(z_{1}, z_{2}\right)=\left(F\left(z_{1}, \bar{z}_{1}, z_{2}, \bar{z}_{2}\right), G\left(z_{1}, \bar{z}_{1}, z_{2}, \bar{z}_{2}\right)\right),
$$

where

$$
\begin{aligned}
F & =z_{1} \sum_{s=\sigma_{n}}^{k} \sum_{j=0}^{3 s+n-2}\left(\alpha_{s j} C^{2 k-2 s+1}+\beta_{s j} D^{2 k-2 s+1}\right) A^{3 s+n-2-j} B^{j} \\
& +\bar{z}_{1} z_{2} \sum_{l=0}^{\omega_{n}} \gamma_{l} D^{2 l} B^{3 k-3 l+n-1},
\end{aligned}
$$

and

$$
\begin{aligned}
G & =z_{2} \sum_{s=\sigma_{n}}^{k} \sum_{j=0}^{3 s+n-2}\left(\tilde{\alpha}_{s j} C^{2 k-2 s+1}+\tilde{\beta}_{s j} D^{2 k-2 s+1}\right) A^{3 s+n-2-j} B^{j} \\
& +z_{1}^{2} \sum_{l=0}^{\omega_{n}} \tilde{\gamma}_{l} C^{2 l} A^{3 k-3 l+n-1} .
\end{aligned}
$$

with $\left.\sigma_{n}:=\mid\right] \frac{2-n}{3}\left[\mid\right.$ and $\left.\omega_{n}:=\llbracket k+\frac{n-1}{3} \mid\right]$

A straightforward calculation yields that

$$
\left[X^{(2)}, Y^{(6 k+2 n)}\right]=I \frac{\partial}{\partial z_{1}}+J \frac{\partial}{\partial z_{2}},
$$


where

$$
I=I_{1}+I_{2}+I_{3} \text {, and } J=J_{1}+J_{2}+I_{3}
$$

with $I_{1}=$

$$
\begin{aligned}
& i z_{1} \sum_{s=\sigma_{n}}^{k-1} C^{2 k-2 s}\left\{\left(2 \tilde{\gamma}_{k-s}+\alpha_{s+1,1}\right) A^{3 s+n}+\right. \\
& +\sum_{j=1}^{3 s+n-1}\left[-2(4 k-s+2-j) \alpha_{s, j-1}+2 \tilde{\alpha}_{s, j-1}+\right. \\
& \left.\left.+(j+1) \alpha_{s+1, j+1}\right] A^{3 s+n-j} B^{j}+(3 s+n+1) \alpha_{s+1,3 s+n+1} B^{3 s+n}\right\}+ \\
& +i z_{1} C^{2 k}\left(2 \mathcal{X}_{1}(n) \tilde{\gamma}_{k} A+\mathcal{X}_{\{0,1\}}(n) \sum_{j=0}^{n}(j+1) \alpha_{1, j+1} A^{n-j} B^{j}\right) \\
& i z_{1} \sum_{s=1}^{k-1} D^{2 k-2 s}\left\{\sum _ { j = 0 } ^ { 3 s + n - 2 } \left[\tilde{\beta}_{s+1, j}-2(3 s+n+2-j) \beta_{s+1, j}+\right.\right. \\
& \left.+(2 k-2 s+1+j) \beta_{s, j}\right] A^{3 s+n-j} B^{j}+\left[\tilde{\beta}_{s+1,3 s+n-1}-2 \beta_{s+1,3 s+n-1}+\right. \\
& \left.\left.+(2 k+s+n) \gamma_{k-s}\right] A B^{3 s+n-1}+\left[\tilde{\beta}_{s+1,3 s+n}-2 \beta_{s+1,3 s+n}\right] B^{3 s+n}\right\}+ \\
& +i z_{1} D^{2 k}\left(\mathcal{X}_{2}(n)\left[\beta_{0,0} A^{2}+\sum_{j=0}^{2}-2(3-j) \beta_{1, j} A^{2-j} B^{j}\right]+\right. \\
& +\mathcal{X}_{\{0,1\}}(n) \sum_{j=0}^{n}-2(n+1-j) \beta_{1, j} A^{n-j} B^{j}+ \\
& \left.+\mathcal{X}_{\{1,2\}}(n)(2 k+n) \gamma_{k} A B^{n-1}+\sum_{j=0}^{n}\left(\tilde{\beta}_{1, j}-2 \beta_{1, j}\right) A^{n-j} B^{j}\right) \\
& I_{2}=i \bar{z}_{1} z_{2} \sum_{s=\sigma_{n}}^{k}\left[2 \tilde{\beta}_{s, 3 s+n-2}-2 \beta_{s, 3 s+n-2}\right] D^{2 k-2 s+1} B^{3 s+n-2} \\
& +
\end{aligned}
$$

and

$$
I_{3}=i f_{1}(A, B)
$$

In a similar way, we write $J=J_{1}+J_{2}+J_{3}$ where 


$$
\begin{aligned}
& J_{1}=i z_{2} \sum_{s=\sigma_{n}}^{k-1} C^{2 k-2 s}\left\{\left[2 \tilde{\alpha}_{s+1,1}-2 \alpha_{s+1,1}-2(4 k-s+n+1) \tilde{\gamma}_{k-s}\right] A^{3 s+n}+\right. \\
& +\sum_{j=1}^{3 s+n-1}\left[-2(4 k-s+1-j) \tilde{\alpha}_{s, j-1}+(j+2) \tilde{\alpha}_{s+1, j+1}-\right. \\
& \left.\left.2 \alpha_{s+1, j+1}\right] A^{3 s+n-j} B^{j}+\left[(3 s+n-2) \tilde{\alpha}_{s+1,3 s+n+1}-2 \alpha_{s+1,3 s+n+1}\right] B^{3 s+n}\right\}+ \\
& +i z_{2} C^{2 k}\left(\mathcal{X}_{\{0,1\}}(n)\left[\sum_{j=0}^{n}\left[(j+2) \tilde{\alpha}_{1, j+1}-2 \alpha_{1, j+1}\right] A^{n-j} B^{j}\right]\right. \\
& \left.+\mathcal{X}_{1}(n)\left[-2(4 k+2) \tilde{\gamma}_{k}\right] A\right)+ \\
& +i z_{2} \sum_{s=\sigma_{n}}^{k-1} D^{2 k-2 s}\left\{\sum _ { j = 0 } ^ { 3 s + n - 2 } \left[(2 k-2 s+2+j) \tilde{\beta}_{s, j}-2 \beta_{s, j}-\right.\right. \\
& \left.-2(3 s+n+1-j) \beta_{s+1, j}\right] A^{3 s+n-j} B^{j}+ \\
& \left.+\left[-2 \gamma_{k-s}-4 \beta_{s+1,3 s+n-1}\right] A B^{3 s+n-1}-2 \beta_{s+1,3 s+n} B^{3 s+n}\right\}+ \\
& +i z_{2} D^{2 k}\left(\mathcal{X}_{1}(n)\left(-2 \gamma_{k}\right) A+\mathcal{X}_{\{0,1\}}(n) \sum_{j=0}^{n}-2(n+1-j) \beta_{1, j} A^{n-j} B^{j}\right) \\
&
\end{aligned}
$$

and

$$
J_{2}=i z_{2}^{2} \sum_{s=\sigma_{n}}^{k}\left(\tilde{\alpha}_{s, 0}-2 \alpha_{s, 0}\right) C^{2 k-2 s+1} A^{3 s+n-1}
$$

and

$$
J_{3}=i z_{2} f_{2}(A, B) .
$$

As before, the elimination of the terms that depend on $C$ and $D$ is equivalent to the solubility of the following system: 


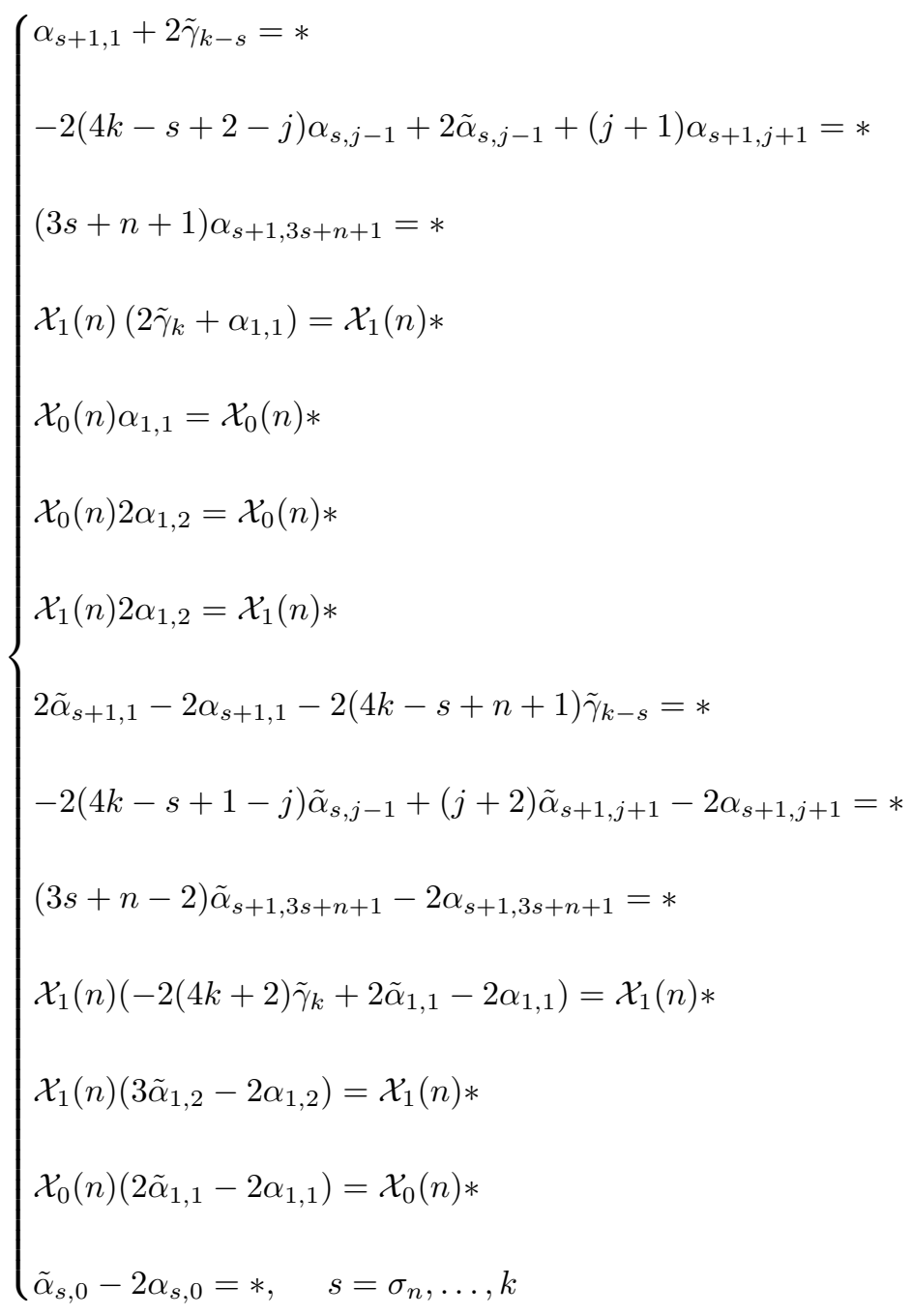

where $s=\sigma_{n}, \ldots, k-1$ and $j=1, \ldots, 3 s+n-1$ (except in the last equation). More, 


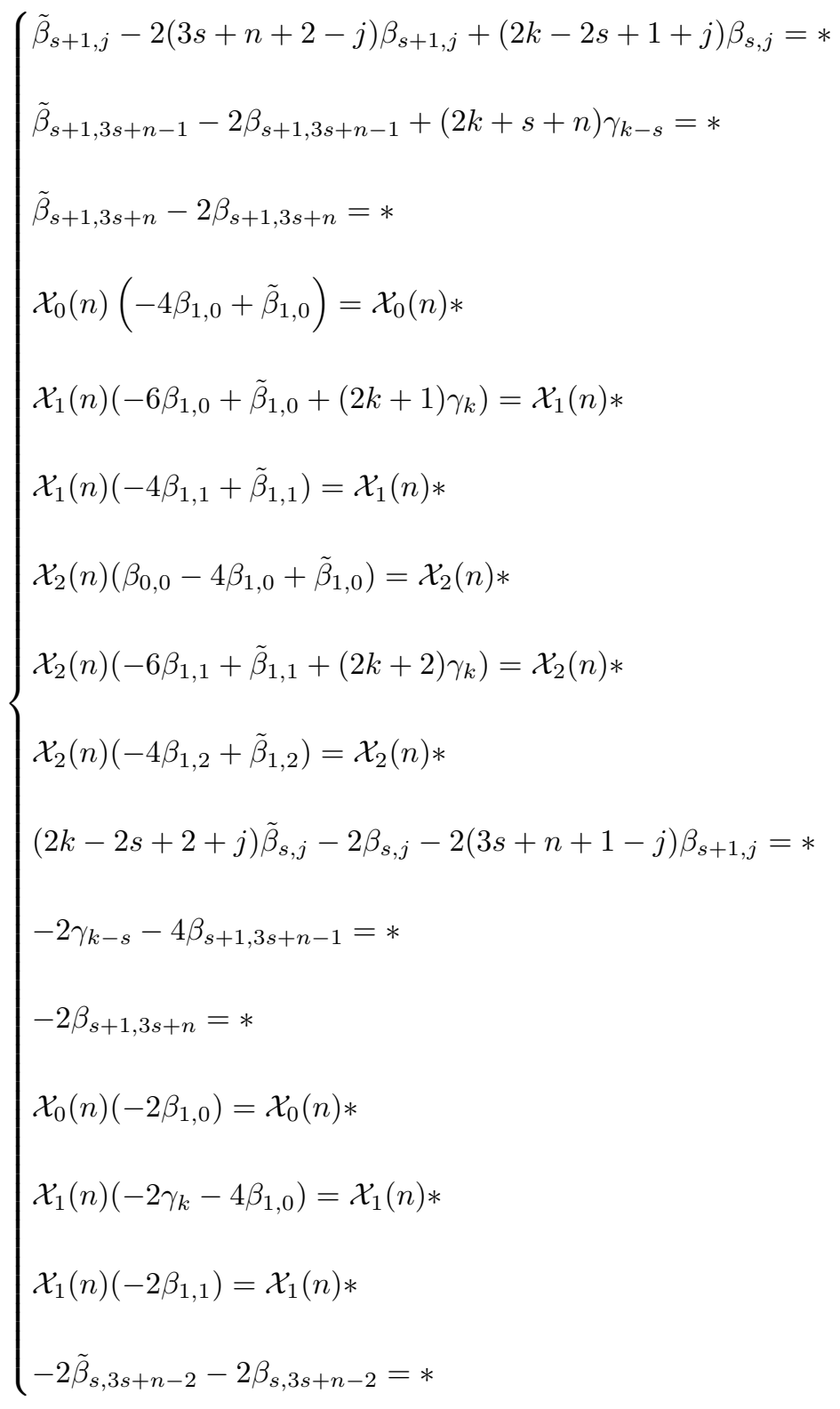

with $s=1, \ldots, k-1$ and $j=0, \ldots, 3 s+n-2$ in the three first equations and $s=\sigma_{n}, \ldots, k-1$ and $j=0, \ldots, 3 s+n-2$ in equations 10,11 and 12 .

The solubility of the last two systems is guaranteed in the same way as done 
for $X^{6 k+2 n}$. Hence the following normal form is obtained:

$$
\tilde{X}^{(6 k+2 n+1)}=i z_{1} \sum_{j=0}^{3 k+n} a_{j} A^{3 k+n-j} B^{j} \frac{\partial}{\partial z_{1}}+i z_{2} \sum_{j=0}^{3 k+n} b_{j} A^{3 k+n-j} B^{j} \frac{\partial}{\partial z_{2}} .
$$

To reduce $\tilde{X}^{(6 k+2 n+1)}$ to the Hamiltonian form given by $H(A, B)$ is enough multiply $\tilde{X}^{(6 k+2 n+1)}$ by

$$
f=1+\sum_{j=0}^{3 k+n+1} \theta_{j} A^{3 k+n+1-j} B^{j}
$$

and choose suitable $\theta_{j}$ as done in the even case.

This finishes the proof of the Theorem 3.5 .

\section{4. $p: q$ resonance: Theorem 3.6}

The proof of formal orbital equivalence in the case of $p: q$ resonance is in essence similar to the proof to the $1: 2$ case (Theorem 3.5), and therefore we shall omit some of the details.

If $X$ is a reversible vector field with $p: q$ resonance in normal form (3.7), then we can use the equalities $\bar{z}_{1}^{q-1} z_{2}^{p} A=z_{1} D, \bar{z}_{1}^{q-1} z_{2}^{p} C=z_{1} A^{q-1} B^{p}, z_{1}^{q} \bar{z}_{2}^{p-1} B=$ $z_{2} C, z_{1}^{q} \bar{z}_{2}^{p-1} D=z_{2} A^{q} B^{p-1}$ and $C D=A^{q} B^{p}$ to rearrange (3.7) into the following form

$$
\begin{aligned}
X= & \left(p i \lambda z_{1}+z_{1} f_{1}(A, B, C, D)+\bar{z}_{1}^{q-1} z_{2}^{p} f_{2}(B, D)\right) \frac{\partial}{\partial z_{1}} \\
& +\left(q i \lambda z_{2}+z_{2} g_{1}(A, B, C, D)+z_{1}^{q} \bar{z}_{2}^{p-1} g_{2}(A, C)\right) \frac{\partial}{\partial z_{2}} .
\end{aligned}
$$

We may rescale the coordinates so that $f_{2}(0,0)=-q$ e $g_{2}(0,0)=p$. Only such coefficients are related with the order $p+q-1$ of the system. The remaining independent terms of $C$ and $D$ have the form $z_{1} A^{m} B^{n} \frac{\partial}{\partial z_{1}}$ and $z_{2} A^{m} B^{n} \frac{\partial}{\partial z_{2}}$ and as before can easily be normalized via a canonical time re-scaling.

We deal now with the dependent terms of $C$ and $D$ having exponent equal to 1 . The order of such terms are at least $p+q+1$.

Consider the terms in $C$ or $D$ whose orders are smaller than $2(p+q)$. They are expressed by:

$$
\begin{aligned}
& z_{1}\left(\alpha_{s, j} C+\beta_{s, j} D\right) A^{s} B^{j} \frac{\partial}{\partial z_{1}} \\
& z_{2}\left(\tilde{\alpha}_{s, j} C+\tilde{\beta}_{s, j} D\right) A^{s} B^{j} \frac{\partial}{\partial z_{2}} \quad, s+j<p+q-1
\end{aligned}
$$

We illustrate this setting by taking the terms of order $p+q+3$ :

$$
\begin{aligned}
& \left(z_{1}\left[\left(\alpha_{1,0} C+\alpha_{1,0} D\right) A+\left(\alpha_{0,1} C+\beta_{0,1} B\right)\right]+\gamma_{o} \bar{z}_{1}^{q-1} z_{2}^{p} B^{2}\right) \frac{\partial}{\partial z_{1}} \\
& \left.\left(z_{2}\left[\tilde{\alpha}_{1,0} C+\tilde{\alpha}_{1,0} D\right) A+\left(\tilde{\alpha}_{0,1} C+\tilde{\beta}_{0,1} B\right)\right]+\tilde{\gamma}_{o} z_{1}^{q} z_{2}^{p-1} A^{2}\right) \frac{\partial}{\partial z_{2}}
\end{aligned} .
$$


The system $X^{(p+q+3)}$ is in the Hamiltonian form provided that its coefficients satisfy some further conditions that are in some sense equivalent to those expressed in 4.25.

The later is achieved via a multiplication by a convenient function. In this way we get, $H^{(p+q+4)}=-i \sum_{j=0}^{2} \alpha_{j}(C-D) A^{2-j} B^{j}$.

In the general case we proceed in a quite similar way. We get then

$$
H=(C-D) h(A, B)
$$

Concerning the elements of order greater than $2(p+q)-1$ two cases must be distinguished:

i) $p+q$ is even. In this case it appears just odd terms given by $X^{(2(p+q) k+2 n+1)}=$ $(I, I I)$, where

$$
\begin{aligned}
I= & z_{1} \sum_{j=0}^{(p+q) k+n} a_{j} A^{(p+q) k+n-j} B^{j}+ \\
& +z_{1} \sum_{s=0}^{k-1} \sum_{j=0}^{(p+q) s+n}\left(f_{s, j} C^{2 k-2 s}+g_{s, j} D^{2 k-2 s}\right) A^{(p+q) s+n-j} B^{j}+ \\
& +\bar{z}_{1}^{q-1} z_{2}^{p} \sum_{l=1}^{k} h_{l} D^{2 l-1} B^{(p+q) k-(p+q) l+n+2-\frac{p+q}{2}}
\end{aligned}
$$

and

$$
\begin{aligned}
I I= & z_{2} \sum_{j=0}^{(p+q) k+n} b_{j} A^{(p+q) k+n-j} B^{j}+ \\
& +z_{2} \sum_{s=0}^{k-1} \sum_{j=0}^{(p+q) s+n}\left(\tilde{f}_{s, j} C^{2 k-2 s}+\tilde{g}_{s, j} D^{2 k-2 s}\right) A^{(p+q) s+n-j} B^{j}+ \\
& +z_{1}^{q} \bar{z}_{2}^{p-1} \sum_{l=1}^{k} \tilde{h}_{l} C^{2 l-1} A^{(p+q) k-(p+q) l+n+1}
\end{aligned}
$$

In the present case the normalization can be done as in the $1: 2-$ case. This means that the independent terms in $C$ and $D$ can be eliminated via canonical re-scaling. The other terms in $C$ and $D$ are, as before, killed via coordinates change.

ii) Finally, if $p+q$ is odd we proceed as above. It is worth to point out that in this case new even terms emerge having a similar form as 4.13 ). This finishes the proof.

\section{Acknowledgements}

This research has been supported in part by EU Marie-Curie IRSES grants Dynamical complex systems (FP7-PEOPLE-2008-IRSES 230844 DYNEURBRAZ) 
and Brazilian-European partnership in Dynamical Systems (FP7-PEOPLE-2012IRSES 318999 BREUDS) as well as EU Marie-Sklodowska-Curie ITN grant Critical Transitions in Complex Systems (H2020-MSCA-2014-ITN 643073 CRITICS). JSWL gratefully acknowledges the support of the EPSRC (GR/A01534/01, GR/S78100/01), FAPESP (2009/18338-2, 2016/50055-4) and Nizhny Novgorod University through grant RNF 14-41-00044. RMM gratefully acknowledges the support of the FAPESP (2015/06903-8).

[1] Arnol'D, V.I., Reversible systems. In: Sagdeev, R.Z.(ed), Nonlinear and Turbulent Processes in Physics 3, Harwood, Chur, pp.1161-1174 (1984).

[2] Arnol'D, V.I., IL'Yashenko, Yu.S., Ordinary differential equations. In: Anosov, D.V., and Arnold, V.I. (ed), Dynamical Systems I, SpringerVerlag (1988).

[3] Arnol'd, V.I., Sevryuk, M.B., Oscillations and bifurcations in reversible systems. In: Sagdeev, R.Z.(ed), Nonlinear Phenomena in Plasma Physics and Hydrodynamics, Mir, Moscow, pp. 31-64 (1986).

[4] Belitskit, G., Smooth equivalence of germs of $C^{\infty}$ of vector fields with one zero or a pair of pure imaginary eigenvalues. Funct. Anal. Appl. 20 (4), 253-259 (1986).

[5] Cicogna G., Gaeta G., Symmetry and Perturbation Theory in Nonlinear Dynamics. Lecture Notes in Physics, vol. 57 (1999).

[6] Devaney, R.L., Reversible diffeomorphisms and flows. Trans. Am. Math. Soc. 218, 89-113 (1976).

[7] Devaney, R.L., Blue sky catestrophes in reversible and Hamiltonian systems. Indiana Univ. Math. J. 26, 247-263 (1977).

[8] Golubitsky,M., Marsden, J.E., Stewart, I., Dellnitz, M., The constrained Liapunov-Schmidt procedure and periodic orbits. Fields Inst. Commun. 4, 81-127 (1995).

[9] ICHIKAWA, F., Finitely determined singularities of formal vector fields. Invent. Math. 66, 199-214 (1982).

[10] ICHIKAWA, F., On finite determinacy of formal vector fields. Invent. Math. 70, 45-52 (1982).

[11] Lamb, J.S.W., Time-reversal symmetry in dynamical systems, PhD thesis, University of Amsterdam (1994).

[12] LAmb, J.S.W., Area-preserving dynamics that is not reversible, Physica A 228, 344-365 (1996).

[13] Lamb, J.S.W., Roberts, J.A.G., Time-reversal symmetry in dynamical systems: a survey. Physica D 112, 1-39 (1998). 
[14] Lamb, J.S.W., Roberts, J.A.G., Capel, H.W., Conditions for local (reversing) symmetries in dynamical systems, Physica A 197, 379-422 (1993).

[15] Martins, R.M., Formal Equivalence Between Normal Forms of Reversible and Hamiltonian Dynamical Systems. Communications in Pure and Applied Analysis, 13(2) (2014) 703-703.

[16] Martins, R.M. Teixeira, M. A., On the Similarity of Hamiltonian and Reversible Vector Fields in 4D. Communications in Pure and Applied Analysis, 10(4) (2011) 1257-1266.

[17] Van der Meer J.C., Sanders, J.A., Vanderbauwhede, A., Hamiltonian structure of the reversible nonsemisimple $1: 1$ resonance. In: Pascal Chossat (ed.), Dynamics, Bifurcation, and Symmetry, New trends and New Tools (1994).

[18] Montgomery, D., Zippin, L., Topological Transformations Groups. Interscience, New York (1955).

[19] Roberts, J.A.G., CAPel, H.W., Area preserving mappings that are not reversible, Phys. Lett. A 162, 243-248 (1992).

[20] Roberts, J.A.G., Quispel, G.R.W. Chaos and time-reversal symmetry. Order and chaos in reversible dynamical systems. Phys. Rep. 216, 63-177 (1992).

[21] Sevryuk, M.B., Reversible Systems. Lecture Notes in Mathematics 1211, Springer, Berlin (1986).

[22] Takens, F., Normal Forms for Certain Singularities of Vector Fields. Ann. Inst. Fourier 23, 163-195 (1973).

[23] Yang, J., Polynomial Normal Forms for Vector Fields. Thesis, Technion (1997). 